\title{
Stable triples, equivariant bundles and dimensional reduction
}

\author{
Steven B. Bradlow* Oscar García-Prada
}

October 29, 2018

\section{Introduction}

The Hitchin-Kobayashi correspondence between stable bundles and solutions to the Hermitian-Einstein equations allows one to apply analytic methods to the study of stable bundles. One such analytic technique, which has not yet been much exploited, is that of dimensional reduction. This is a useful tool for studying certain special solutions to partial differential equations; in particular it is useful for studying solutions which are invariant under the action of some symmetry group. When applied to the Hermitian-Einstein equations, it thus provides a way of looking at holomorphic bundle structures which are both stable and invariant under some group action on the bundle, i.e. of looking at equivariant stable bundles.

The main idea in dimensional reduction is the following: Suppose we have a partial differential equation defined on a space which has a symmetry, i.e. which supports some group action. Then by integrating over the group orbits, any solution which is invariant under the group action becomes an object defined in a space of lower dimension than that of the original setting for the general solutions. This lower dimensional space is the orbit space of the group action, and in that space the special solutions to the original equations can be re-interpreted as ordinary solutions to some new set of equations.

In particular, suppose that we start with a 4 -manifold, $M$, a Lie group $G$ which acts on it, and a complex vector bundle $E$ to which this action lifts. In such a situation, there can be equivariant solutions to the Hermitian-Einstein equations, and dimensional reduction can be applied. The orbit space $E / G$ will be a new bundle over $M / G$, the orbit space of the group action on the original 4-manifold. The equivariant solutions to the original Hermitian-Einstein equations will be solutions to a new set of equations on the bundle $E / G \longrightarrow M / G$. For example, the equations introduced by Hitchin in [H], namely the Anti-Self-Dual equations on a Riemann surface, can be viewed as arising in this way.

\footnotetext{
${ }^{*}$ Supported in part by a NSF-NATO Postdoctoral Fellowship and NSF grant DMS 93-03545
} 
A special case of the above situation occurs when the 4-manifold $M$ is a complex surface, and the orbit space $M / G$ also admits a complex structure. Solutions to the Hermitian-Einstein equations then correspond to stable holomorphic structures on the bundle $E$. In such a situation, dimensional reduction acquires an extra, holomorphic interpretation. It results in information about the equivariant stable bundles on $M$ being encoded in a holomorphic interpretation for the dimensionally reduced equations on $M / G$.

These sort of ideas are developed in [GP3], where they are applied to certain $S U(2)$-equivariant bundles over $X \times P^{1}$. Here $X$ is a closed Riemann surface and the $S U(2)$-action is trivial on $X$ and the standard one on $P^{1}$. In this case, the equivariant holomorphic bundles over $X \times P^{1}$ correspond to holomorphic pairs (i.e. bundles plus prescribed global sections) over $X$. The dimensional reduction of the HermitianEinstein equations gives the vortex equations, and the stable equivariant bundles on $X \times P^{1}$ correspond (by dimensional reduction) to $\tau$-stable holomorphic pairs on $X$, with $\tau$-stability as defined in [B2] and GP3].

However not all the $S U(2)$-equivariant holomorphic bundles over $X \times P^{1}$ correspond to holomorphic pairs on $X$. In fact those that do form a rather restricted subset of the set of all such equivariant bundles. A very natural relaxation of this restriction leads to a class of equivariant bundles on $X \times P^{1}$ which still corresponds to data on the (lower dimensional) space $X$, but not necessarily to holomorphic pairs. What such bundles correspond to is a pair of bundles plus a holomorphic homomorphism between them. We call such data a holomorphic triple.

In this paper we undertake a detailed investigation of holomorphic triples over the closed Riemann surface $X$. In particular, we define, in Section 3, a notion of stability for such objects. We explore the relationship between the stability of a triple and the stability of the corresponding equivariant bundle over $X \times P^{1}$. An important feature of the definition is that, like in the case of holomorphic pairs, it involves a real parameter. This can be traced back to the fact that the definition of stability for a bundle over $X \times P^{1}$ depends on the polarization (choice of Kähler metric) on $X \times P^{1}$. We discuss the nature of this parameter, and its influence on the properties of the stable triples. We show for example that

In all cases, with one exception, the parameter in the definition of triples stability lies in a bounded interval. The interval is partitioned by a finite set of non-generic values.

Our main result is given in Section 4. Loosely speaking, it is that the stable triples over $X$ can be considered the dimensional reduction of the stable equivariant bundles over $X \times P^{1}$. In other words,

A holomorphic triple over $X$ is stable if and only if the corresponding $S U(2)$-equivariant extension over $X \times P^{1}$ is stable

In GP3 dimensional reduction is applied to the Hermitian-Einstein equation on equivariant bundles over $X \times P^{1}$. The result is that on bundles corresponding to triples over $X$, the equivariant solutions correspond to solutions to a pair of Coupled Vortex 
Equations on the two bundles in the triple. By combining this result, our dimensional reduction result for stable bundles, and the Hitchin-Kobayashi correspondence, we can thus show

There is a Hitchin-Kobayashi correspondence between stability of a triple and existence of solutions to the Coupled Vortex Equations.

This is discussed in Section 5. In Section 6 we discuss the moduli spaces of stable triples. By identifying these as fixed point sets of an $S U(2)$-action on the moduli spaces of stable bundles over $X \times P^{1}$, we obtain results such as

For fixed value of the stability parameter, the moduli space of stable triples is a quasi- projective variety. For generic values of the parameter, and provided the ranks and degrees of the two bundles satisfy a certain coprimality condition, the moduli space is projective

In Section 2 we have collected together the basic definitions and background material that we will need.

\section{Background and Preliminaries}

\subsection{Basic Definitions}

Let $X$ be a compact Riemann surface.

Definition 2.1 A holomorphic triple on $X$ is a triple $\left(E_{1}, E_{2}, \Phi\right)$ consisting of two holomorphic vector bundles $E_{1}$ and $E_{2}$ on $X$ together with a homomorphism $\Phi: E_{2} \longrightarrow$ $E_{1}$, i.e. an element $\Phi \in H^{0}\left(\operatorname{Hom}\left(E_{2}, E_{1}\right)\right)$.

In this paper we will develop a theory of holomorphic triples as objects in their own right. We will also show how they arise from $S U(2)$-equivariant holomorphic vector bundles over $X \times P^{1}$.

Let $S U(2)$ act on $X \times P^{1}$ trivially on $X$ and in the standard way on $P^{1}$, that is we regard $P^{1}$ as the homogeneous space $S U(2) / U(1)$. Let $F$ be a $C^{\infty}$ complex vector bundle over $X \times P^{1}$.

Definition 2.2 The bundle $F$ is said to be $S U(2)$-equivariant if there is an action of $S U(2)$ on $F$ covering the action on $X \times P^{1}$. Similarly a holomorphic vector bundle $F$ is $S U(2)$-equivariant if it is $S U(2)$-equivariant as a $C^{\infty}$ bundle and in addition the action of $S U(2)$ on $F$ is holomorphic.

\subsection{Smooth and Holomorphic Equivariant bundles}

Our main objective in this section will be the study of $S U(2)$-equivariant holomorphic vector bundles on $X \times P^{1}$, however, before addressing this, we shall analyse the much 
easier problem of classifying the $S U(2)$-equivariant $C^{\infty}$ ones. Let $p$ and $q$ be the projections from $X \times P^{1}$ to the first and second factors respectively.

Proposition 2.3 Every $S U(2)$-equivariant $C^{\infty}$ vector bundle $F$ over $X \times P^{1}$ can be equivariantly decomposed, uniquely up to isomorphism, as

$$
F=\bigoplus_{i} p^{*} E_{i} \otimes q^{*} H^{\otimes n_{i}}
$$

where $E_{i}$ is a $C^{\infty}$ vector bundle over $X, H$ is the $C^{\infty}$ line bundle over $P^{1}$ with Chern class 1 , and $n_{i} \in Z$ are all different.

Proof. See GP3, Proposition 3.1].

We shall describe now $S U(2)$-invariant holomorphic structures on a fixed $S U(2)$ equivariant $C^{\infty}$ vector bundle over $X \times P^{1}$. We shall restrict ourselves, however, to the case which is relevant in connection to holomorphic triples. Let $E_{1}$ and $E_{2}$ be $C^{\infty}$ vector bundles on $X$ and let $H$ be as in Proposition 2.3. Consider the $S U(2)$-equivariant $C^{\infty}$ vector bundle

$$
F=p^{*} E_{1} \oplus p^{*} E_{2} \otimes q^{*} H^{\otimes 2},
$$

Note that the total space of $p^{*} E_{1}$ is $E_{1} \times P^{1}$, and the action of $S U(2)$ that we are considering is trivial on $E_{1}$ and the standard one on $P^{1}$; similarly for $p^{*} E_{2}$. On the other hand, recall that the $S U(2)$-equivariant line bundle $H^{\otimes 2}$ over $P^{1} \cong S U(2) / U(1)$ corresponds to the one dimensional representations of $U(1)$ given by $e^{i 2 \alpha}$, i.e. $H^{\otimes 2}=$ $S U(2) \times_{U(1)} C$, where $(g, v) \sim\left(g^{\prime}, v^{\prime}\right)$ if there is an $e^{i \alpha} \in U(1)$ such that $g^{\prime}=e^{-i \alpha} g$ and $v^{\prime}=e^{i 2 \alpha} v$.

The action of $S U(2)$ on $S U(2) \times C$, given by

$$
\gamma \cdot(g, v)=(\gamma g, v) \quad \text { for } \quad \gamma \in S U(2) \text { and }(g, v) \in S U(2) \times C,
$$

descends to an action on $H^{\otimes 2}$.

In order to avoid the introduction of more notation we shall denote a $C^{\infty}$ vector bundle and the same bundle endowed with a holomorphic structure by the same symbol. The distinction will be made explicit unless it is obvious from the context.

\subsection{Dimensional Reduction of Bundles}

Proposition 2.4 Every $S U(2)$-equivariant holomorphic vector bundle $F$ with underlying $S U(2)$-equivariant $C^{\infty}$ structure given by (1) is in one-to-one correspondence with a holomorphic extension of the form

$$
0 \longrightarrow p^{*} E_{1} \longrightarrow F \longrightarrow p^{*} E_{2} \otimes q^{*} \mathcal{O}(2) \longrightarrow 0,
$$

where $E_{1}$ and $E_{2}$ are the bundles over $X$ defining (1) equipped with holomorphic structures.

Moreover, every such extension is defined by an element $\Phi \in \operatorname{Hom}\left(E_{2}, E_{1}\right)$, and is thus in one-to-one correspondence with the holomorphic triple $\left(E_{1}, E_{2}, \Phi\right)$ on $X$. 
Proof. We shall give here only a brief sketch of the proof (see [GP3, Proposition 3.9] for details). Let $E_{1}$ and $E_{2}$ be two holomorphic vector bundles over $X$. The extensions over $X \times P^{1}$ of the form (2) are parametrized by

$$
H^{1}\left(X \times P^{1}, p^{*}\left(E_{1} \otimes E_{2}^{*}\right) \otimes q^{*} \mathcal{O}(-2)\right) ;
$$

but this is isomorphic to

$$
H^{0}\left(X, E_{1} \otimes E_{2}^{*}\right) \otimes H^{1}\left(P^{1}, \mathcal{O}(-2)\right) \cong H^{0}\left(X, E_{1} \otimes E_{2}^{*}\right),
$$

by means of the Künneth formula, the fact that $H^{0}\left(P^{1}, \mathcal{O}(-2)\right)=0$ and

$$
H^{1}\left(P^{1}, \mathcal{O}(-2)\right) \cong H^{0}\left(P^{1}, \mathcal{O}\right)^{*} \cong C .
$$

Therefore after fixing an element in $H^{1}\left(P^{1}, \mathcal{O}(-2)\right)$, the homomorphism $\Phi$ can be identified with the extension class defining $F$.

Certainly, since the action of $S U(2)$ on the extension class is trivial (note that this action is induced from the action on $E_{1} \otimes E_{2}^{*}$, which is trivial), then the bundle $F$ defined by the triple $\left(E_{1}, E_{2}, \Phi\right)$ is an $S U(2)$-equivariant holomorphic vector bundle. One can show with a little bit more work that in fact every $S U(2)$-equivariant holomorphic structure on the $S U(2)$-equivariant $C^{\infty}$ bundle (四) defines an extension of the form (2).

What this Proposition says is that holomorphic triples over $X$ can be regarded as a "dimensional reduction" of certain $S U(2)$-equivariant holomorphic vector bundles over $X \times P^{1}$.

\subsection{Subtriples}

Definition 2.5 A triple $T^{\prime}=\left(E_{1}^{\prime}, E_{2}^{\prime}, \Phi^{\prime}\right)$ is a subtriple of $T=\left(E_{1}, E_{2}, \Phi\right)$ if

(1) $E_{i}^{\prime}$ is a coherent subsheaf of $E_{i}$, for $i=1,2$

(2) $\Phi^{\prime}=\left.\Phi\right|_{E_{2}}$, i.e. $\Phi^{\prime}$ is the restriction of $\Phi$.

In other words we have the commutative diagram

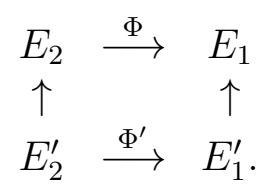

If $E_{1}^{\prime}=E_{2}^{\prime}=0$, the subtriple is called the trivial subtriple.

Remark. When studying stability criteria, it will suffice, as usual, to consider saturated subsheaves, that is subsheaves whose quotient sheaves are torsion free. On a Riemann surface these are precisely subbundles. 
With this definition, subobjects of the triple $\left(E_{1}, E_{2}, \Phi\right)$ are related to subsheaves of the corresponding $S U(2)$-equivariant bundle $F \longrightarrow X \times P^{1}$ in an appropriate way. First note that the correspondence between triples on $X$ and bundles on $X \times P^{1}$ can be extended more generally to arbitrary coherent sheaves. Namely, if $S_{1}$ and $S_{2}$ are two coherent sheaves on $X$ and $\Psi \in \operatorname{Hom}\left(S_{2}, S_{1}\right)$ the triple $\left(S_{1}, S_{2}, \Psi\right)$ defines a coherent sheaf $U$ over $X \times P^{1}$. This sheaf is given, as for bundles, as an extension

$$
0 \longrightarrow p^{*} S_{1} \longrightarrow U \longrightarrow p^{*} S_{2} \otimes \mathcal{O}(2) \longrightarrow 0 \text {. }
$$

The proof is very much as for the case of bundles, once we have fixed $S_{1}$ and $S_{2}$, the extensions as (3) are parametrized by

$$
\operatorname{Ext}_{X \times P^{1}}^{1}\left(p^{*} S_{2} \otimes q^{*} \mathcal{O}(2), p^{*} S_{1}\right) .
$$

But, by the Künneth formula for the Ext groups, this group is isomorphic to

$$
\operatorname{Hom}_{X}\left(S_{2}, S_{1}\right) \otimes \operatorname{Ext}_{P^{1}}^{1}(\mathcal{O}, \mathcal{O}(2)) \oplus \operatorname{Ext}_{X}^{1}\left(S_{2}, S_{1}\right) \otimes \operatorname{Hom}_{P^{1}}(\mathcal{O}(2), \mathcal{O}) .
$$

This reduces to $\operatorname{Hom}_{X}\left(S_{2}, S_{1}\right)$, since $\operatorname{Hom}_{P^{1}}(\mathcal{O}(2), \mathcal{O}) \cong H^{0}\left(P^{1}, \mathcal{O}(-2)\right)=0$ and

$$
\operatorname{Ext}_{P^{1}}^{1}(\mathcal{O}, \mathcal{O}(2)) \cong H^{1}\left(P^{1}, \mathcal{O}(-2)\right) \cong C .
$$

Lemma 2.6 Let $F \longrightarrow X \times P^{1}$ be the bundle associated to a triple $\left(E_{1}, E_{2}, \Phi\right)$. Then every $S U(2)$-invariant coherent subsheaf $F^{\prime} \subset F$ is an extension of the form

$$
0 \longrightarrow p^{*} E_{1}^{\prime} \longrightarrow F^{\prime} \longrightarrow p^{*} E_{2}^{\prime} \otimes q^{*} \mathcal{O}(2) \longrightarrow 0,
$$

with $E_{1}^{\prime} \subset E_{1}$ and $E_{2}^{\prime} \subset E_{2}$ coherent subsheaves, making the following diagram commutative

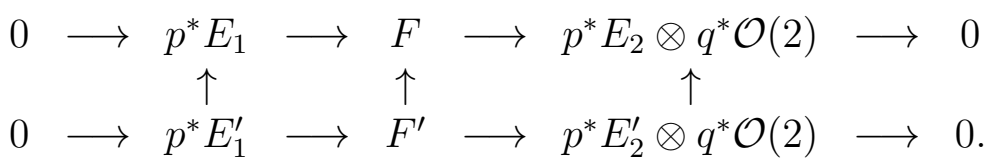

Thus $F^{\prime}$ corresponds to a triple $\left(E_{1}^{\prime}, E_{2}^{\prime}, \Phi^{\prime}\right)$, for $\Phi^{\prime} \in \operatorname{Hom}\left(E_{2}^{\prime}, E_{1}^{\prime}\right)$.

Proof. Let $f: F^{\prime} \rightarrow p^{*} E_{2} \otimes q^{*} \mathcal{O}(2)$ be the composition of the injection $F^{\prime} \rightarrow F$ with the surjective map $F \rightarrow p^{*} E_{2} \otimes q^{*} \mathcal{O}(2)$. Consider the commutative diagram

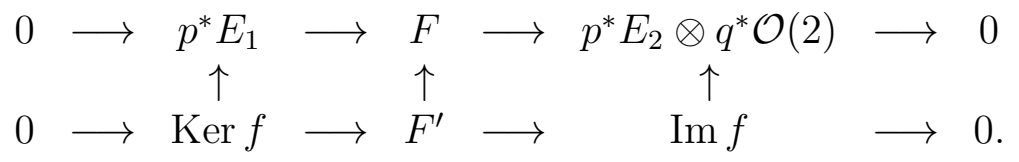

The $S U(2)$-invariance of $F^{\prime}$ implies that of $\operatorname{Ker} f$ and $\operatorname{Im} f$. It suffices therefore to show that if $E$ is a holomorphic vector bundle over $X$ and if $p^{*} E$ is the pull-back to $X \times P^{1}$, then every $S U(2)$-invariant subsheaf of $p^{*} E$ is isomorphic to a sheaf of the form $p^{*} E^{\prime}$ for $E^{\prime}$ a subsheaf of $E$. Indeed, the action of $S U(2)$ on $p^{*} E$ can be extended to an action of $S L(2, C)$. Let $F^{\prime} \subset p^{*} E$ be a $S L(2, C)$-invariant coherent 
subsheaf. Consider the action of a subgroup $C^{*} \subset S L(2, C)$ on $X \times C \subset X \times P^{1}$ and let $A=H^{0}(X, E)$ be the space of global sections. Clearly $H^{0}\left(X \times C, F^{\prime}\right) \subset A[t]$, that is, $H^{0}\left(X \times C, F^{\prime}\right)=\bigoplus_{k=0}^{N} B_{k}$, where an element of $B_{k}$ is of the form $s t^{k}$ for $s \in A$. The action of $\alpha \in C^{*}$ is given by

$$
\alpha\left(s t^{k}\right)=s \alpha^{k} t^{k} .
$$

By choosing another subgroup $C^{*} \subset S L(2, C)$, the $S L(2, C)$-invariance of $F^{\prime}$ implies that $H^{0}\left(X \times C, F^{\prime}\right)=B_{0}$ and hence $F^{\prime}=p^{*} E^{\prime}$ for $E^{\prime} \subset E$ a coherent subsheaf.

We shall show in the next lemma that the triple associated to $F^{\prime} \subset F$ is in fact a subtriple of $\left(E_{1}, E_{2}, \Phi\right)$, and conversely, every subtriple of $\left(E_{1}, E_{2}, \Phi\right)$ defines a unique $S U(2)$-invariant coherent subsheaf of $F$.

Lemma 2.7 Let $E_{1}^{\prime} \subset E_{1}$ and $E_{2}^{\prime} \subset E_{2}$ be coherent subsheaves and let $\Phi^{\prime} \in \operatorname{Hom}\left(E_{2}^{\prime}, E_{1}^{\prime}\right)$. Let $F^{\prime}$ be the coherent sheaf over $X \times P^{1}$ defined by the triple $\left(E_{1}^{\prime}, E_{2}^{\prime}, \Phi^{\prime}\right)$. Then $F^{\prime}$ is a subsheaf of $F$ making the diagram

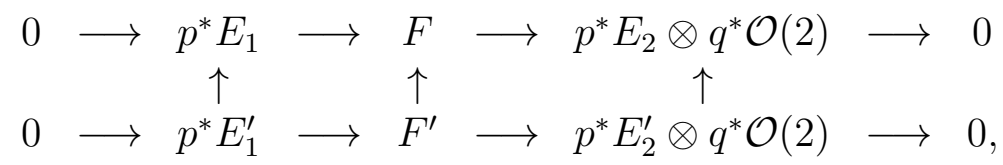

commutative if and only if $\left(E_{1}^{\prime}, E_{2}^{\prime}, \Phi^{\prime}\right)$ is a subtriple of $\left(E_{1}, E_{2}, \Phi\right)$.

Proof. Consider the diagram

$$
\operatorname{Hom}\left(E_{2}^{\prime}, E_{1}^{\prime}\right) \stackrel{i}{\longrightarrow} \operatorname{Hom}\left(E_{2}^{\prime}, E_{1}\right) \stackrel{j}{\longleftarrow} \operatorname{Hom}\left(E_{2}, E_{1}\right) .
$$

To say that $\left(E_{1}^{\prime}, E_{2}^{\prime}, \Phi^{\prime}\right)$ is a subtriple of $\left(E_{1}, E_{2}, \Phi\right)$ is equivalent to saying that

$$
i\left(\Phi^{\prime}\right)=j(\Phi) \text {. }
$$

Under the isomorphisms

$$
\begin{aligned}
& \operatorname{Hom}\left(E_{2}^{\prime}, E_{1}^{\prime}\right) \cong \operatorname{Ext}^{1}\left(p^{*} E_{1}^{\prime}, p^{*} E_{2}^{\prime} \otimes q^{*} \mathcal{O}(2)\right) \\
& \operatorname{Hom}\left(E_{2}^{\prime}, E_{1}\right) \cong \operatorname{Ext}^{1}\left(p^{*} E_{1}, p^{*} E_{2}^{\prime} \otimes q^{*} \mathcal{O}(2)\right) \\
& \operatorname{Hom}\left(E_{2}, E_{1}\right) \cong \operatorname{Ext}^{1}\left(p^{*} E_{1}, p^{*} E_{2} \otimes q^{*} \mathcal{O}(2)\right),
\end{aligned}
$$

$i\left(\Phi^{\prime}\right)$ defines an extension $\tilde{F}^{(i)}$ which makes the following diagram commutative

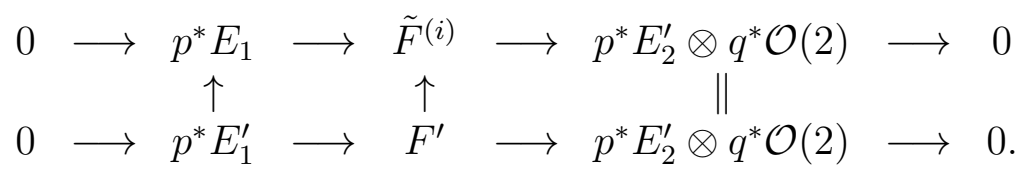

In particular $F^{\prime}$ is a subsheaf of $\tilde{F}^{(i)}$. On the other hand $j(\Phi)$ defines an extension $\tilde{F}^{(j)}$ which fits in the following commutative diagram

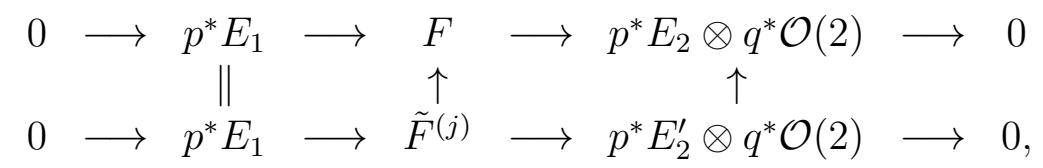

and in particular $\tilde{F}^{(j)}$ is a subsheaf of $F$. Since $i\left(\Phi^{\prime}\right)=j(\Phi), \tilde{F}^{(i)} \cong \tilde{F}^{(j)}$ and we can compose the above two diagrams to obtain the desired result. 


\subsection{Simple Triples}

Definition 2.8 Let

$$
H^{0}\left(E_{1}, E_{2}, \Phi\right)=\left\{(u, v) \in H^{0}\left(\text { End } E_{1}\right) \oplus H^{0}\left(\text { End } E_{2}\right) \mid u \Phi=\Phi v\right\} .
$$

We say a holomorphic triple $\left(E_{1}, E_{2}, \Phi\right)$ is simple if $H^{0}\left(E_{1}, E_{2}, \Phi\right) \simeq C$, i.e. if the only elements in $H^{0}\left(E_{1}, E_{2}, \Phi\right)$ are of the form $\lambda\left(I_{1}, I_{2}\right)$ where $\lambda$ is a constant and $\left(I_{1}, I_{2}\right)$ denote the identity maps on $E_{1}$ and $E_{2}$.

This definition too is dictated by the correspondence between triples on $X$ and equivariant holomorphic extensions over $X \times P^{1}$ :

Proposition 2.9 The triple $\left(E_{1}, E_{2}, \Phi\right)$ is simple if and only if the $S U(2)$-equivariant bundle $F$ associated to $\left(E_{1}, E_{2}, \Phi\right)$ is $S U(2)$-equivariantly simple.

Proof. The definition of simplicity for an equivariant bundle is the obvious generalization of that for an ordinary holomorphic bundle. Namely, an equivariant bundle is said to be equivariantly simple if it has no other invariant endomorphisms than the constant multiples of the identity. The proof of the Proposition follows from the following lemma.

Lemma 2.10 Let $T=\left(E_{1}, E_{2}, \Phi\right)$ and $T^{\prime}=\left(E_{1}^{\prime}, E_{2}^{\prime}, \Phi^{\prime}\right)$ be two holomorphic triples over $X$ and $F$ and $F^{\prime}$ be the corresponding $S U(2)$-equivariant holomorphic vector bundles over $X \times P^{1}$. Then, every $S U(2)$-equivariant homomorphism $g: F \longrightarrow F^{\prime}$ induces homomorphisms $u: E_{1} \longrightarrow E_{1}^{\prime}$ and $v: E_{2} \longrightarrow E_{2}^{\prime}$ such that

$$
u \Phi=\Phi^{\prime} v
$$

Conversely, given morphisms $u$ and $v$ satisfying (8) there exists a unique morphism $g: F \longrightarrow F^{\prime}$ inducing $u$ and $v$.

Proof. The map $g$ can be decomposed as

$$
g=\left(\begin{array}{ll}
g_{1} & f_{1} \\
f_{2} & g_{2}
\end{array}\right)
$$

where $g_{1}: p^{*} E_{1} \longrightarrow p^{*} E_{1}^{\prime}, g_{2}: p^{*} E_{2} \otimes q^{*} \mathcal{O}(2) \longrightarrow p^{*} E_{2}^{\prime} \otimes q^{*} \mathcal{O}(2), f_{1}: p^{*} E_{2} \otimes q^{*} \mathcal{O}(2) \longrightarrow$ $p^{*} E_{1}^{\prime}$ and $f_{2}: p^{*} E_{1} \longrightarrow p^{*} E_{2}^{\prime} \otimes q^{*} \mathcal{O}(2)$. By invariance it is very easy to see (cf. [GP3, Proposition 3.9]) that $g_{1}=p^{*} u, g_{2}=p^{*} v$, and $f_{1}=0=f_{2}$. Equation (8) follows from the commutativity of the following diagram

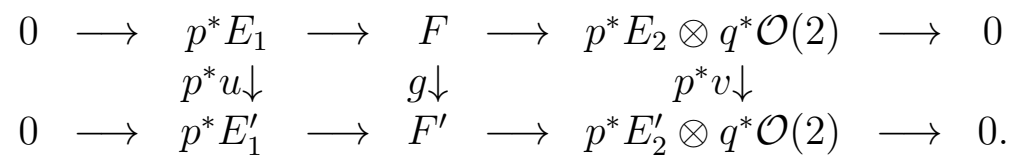


From this lemma it follows that every $S U(2)$-invariant endomorphism of $F$ induces endomorphisms $u: E_{1} \longrightarrow E_{1}$ and $v: E_{2} \longrightarrow E_{2}$, satisfying $u \Phi=\Phi v$. And conversely, given endomorphisms $u$ and $v$ satisfying $u \Phi=\Phi v$ there exists a unique endomorphism of $F$ inducing $u$ and $v$.

The definition of simplicity for a triple given above is also motivated by a deformation theory description of the "tangent space" to the space of triples. We will say more about this in Section 6 .

\subsection{Reducible Triples}

A related, but inequivalent, notion to simplicity is that of irreducibility. We make the following definitions.

Definition 2.11 We say the triple $T=\left(E_{1}, E_{2}, \Phi\right)$ is reducible if there are direct sum decompositions $E_{1}=\bigoplus_{i=1}^{n} E_{1 i}, E_{2}=\bigoplus_{i=1}^{n} E_{2 i}$, and $\Phi=\bigoplus_{i=1}^{n} \Phi_{i}$, such that $\Phi_{i} \in \operatorname{Hom}\left(E_{2 i}, E_{1 i}\right)$. We adopt the convention that if $E_{2 i}=0$ or $E_{1 i}=0$ for some $i$, then $\Phi_{i}$ is the zero map. With $T_{i}=\left(E_{1 i}, E_{2 i}, \Phi_{i}\right)$, we write $T=\bigoplus_{i=1}^{n} T_{i}$. Thus $T$ is reducible if it has a decomposition as a direct sum of subtriples.

If $T$ is not reducible, we say $T$ is irreducible.

Proposition 2.12 If a triple $T=\left(E_{1}, E_{2}, \Phi\right)$ is simple, then it is irreducible.

Proof. Suppose $T$ is reducible, with $T=\bigoplus_{i=1}^{n} T_{i}$. Then we can define $(u, v) \in$ $H^{0}\left(E_{1}, E_{2}, \Phi\right)$ by $u=\bigoplus_{i=1}^{n} \lambda_{i} I_{1 i}, v=\bigoplus_{i=1}^{n} \lambda_{i} I_{2 i}$, where for each $i, \lambda_{i} \in C$ and $I_{1 i}\left(I_{2 i}\right)$ is the identity map on $E_{1 i}\left(E_{2 i}\right)$. Clearly $T$ is not simple.

Proposition 2.13 A holomorphic triple $T=\left(E_{1}, E_{2}, \Phi\right)$ over $X$ is irreducible if and only if the corresponding $S U(2)$-equivariant extension $F \longrightarrow X \times P^{1}$ is equivariantly irreducible, i.e. cannot be decomposed as a sum of $S U(2)$-equivariant extensions of the form (4).

Proof. This follows directly from the relation between subtriples of $T$ and $S U(2)$-equivariant subbundles of $F$ (cf. Lemmas 2.6 and 2.7).

\subsection{Equations for special Metrics}

Given a holomorphic vector bundle over a compact Riemann surface there is a natural condition for a Hermitian metric on it: that of being projectively flat. By choosing a metric on $X$ one can rewrite this condition in a way which turns out to be the right generalization for higher dimensional manifolds: the Hermitian-Einstein condition. 
Since we shall use this notion on $X$ as well as on $X \times P^{1}$, we shall define it on a compact Kähler manifold of arbitrary dimension $(M, \omega)$.

Let $E$ be a holomorphic vector bundle over $M$ and $h$ be a Hermitian metric on $E$. Recall that there is on $E$ a unique connection compatible with both the metric and the holomorphic structure - the so-called metric connection. Let $F_{h}$ be its curvature and $\Lambda F_{h}$ be the contraction of $F_{h}$ with the Kähler form $\omega . \Lambda F_{h}$ is hence a smooth section of End $E$. The metric $h$ is said to be Hermitian-Einstein with respect to $\omega$ if

$$
\sqrt{-1} \Lambda F_{h}=\lambda I_{E}
$$

where $I_{E} \in \Omega^{0}$ (End $\left.E\right)$ is the identity and $\lambda$ is a constant which is determined by integrating the trace of (9). Using that the degree of $E$, defined as

$$
\operatorname{deg} E=\frac{1}{(m-1) !} \int_{M} c_{1}(E) \wedge \omega^{m-1},
$$

where $m$ is the dimension of $M$ and $c_{1}(E)$ is the first Chern class of $E$, given via Chern-Weil theory by

$$
\operatorname{deg} E=\frac{i}{2 \pi} \int_{M} \operatorname{Tr}\left(\Lambda F_{h}\right) \frac{\omega^{m}}{m !}
$$

we obtain

$$
\lambda=\frac{2 \pi}{\operatorname{Vol} M} \frac{\operatorname{deg} E}{\operatorname{rank} E} .
$$

Coming back to our compact Riemann surface $X$, let us choose a metric on $X$ with Kähler form $\omega_{X}$ and volume normalized to one. Given a holomorphic triple $\left(E_{1}, E_{2}, \Phi\right)$ on $X$ it was shown in GP3 that there are natural equations for metrics on the bundles $E_{1}$ and $E_{2}$. These equations, formally similar to the Hermitian-Einstein equations, involve in a natural way the endomorphism $\Phi$. If $E_{1}$ and $E_{2}$ are endowed with Hermitian metrics one can form smooth sections of End $E_{1}$ and End $E_{2}$ respectively by taking the compositions $\Phi \Phi^{*}$ and $\Phi^{*} \Phi$. Here $\Phi^{*}$ is the adjoint of $\Phi$ with respect to the metrics of $E_{1}$ and $E_{2}$. The equations for the metrics $h_{1}$ and $h_{2}$ on $E_{1}$ and $E_{2}$, respectively, are given by

$$
\left.\begin{array}{l}
\sqrt{-1} \Lambda F_{h_{1}}+\Phi \Phi^{*}=2 \pi \tau I_{E_{1}} \\
\sqrt{-1} \Lambda F_{h_{2}}-\Phi^{*} \Phi=2 \pi \tau^{\prime} I_{E_{2}}
\end{array}\right\}
$$

where $\tau$ and $\tau^{\prime}$ are real parameters.

We first observe that, in order to solve (10), the parameters $\tau$ and $\tau^{\prime}$ must be related. Indeed, by adding the trace of the two equations in (10), and since $\operatorname{Tr}\left(\Phi \Phi^{*}\right)=\operatorname{Tr}\left(\Phi^{*} \Phi\right)$, we get

$$
\sqrt{-1} \operatorname{Tr}\left(\Lambda F_{h_{1}}\right)+\sqrt{-1} \operatorname{Tr}\left(\Lambda F_{h_{2}}\right)=2 \pi r_{1} \tau+2 \pi r_{2} \tau^{\prime}
$$

where $r_{1}$ and $r_{2}$ are the ranks of $E_{1}$ and $E_{2}$ respectively. By integrating this equation and, since

$$
\operatorname{deg} E_{1}=\frac{\sqrt{-1}}{2 \pi} \int_{X} \operatorname{Tr}\left(\Lambda F_{h_{1}}\right) \omega \quad \text { and } \quad \operatorname{deg} E_{2}=\frac{\sqrt{-1}}{2 \pi} \int_{X} \operatorname{Tr}\left(\Lambda F_{h_{2}}\right) \omega,
$$


we obtain

$$
r_{1} \tau+r_{2} \tau^{\prime}=\operatorname{deg} E_{1}+\operatorname{deg} E_{2} .
$$

There is therefore just one independent parameter, that we choose to be $\tau$. These equations are called the coupled $\tau$-vortex equations by analogy with the vortex equations on a single bundle studied in [B1, B2, GP1, GP2].

\subsection{Dimensional Reduction of Equations}

The coupled vortex equations have similar interpretations to the Hermitian-Einstein equation and the vortex equations on a single bundle. They can be interpreted both as the equations satisfied by the minima of a certain gauge-theoretical functional - a generalized Yang-Mills-Higgs-type functional-as well as moment map equations in the sense of symplectic geometry (see GP3, Section 2] for details). In fact, the relation between the coupled vortex equations and the Hermitian-Einstein equation that we shall exploit here is of a more intimate nature. Namely, the coupled vortex equations are a dimensional reduction of the Hermitian-Einstein equation under the action of $S U(2)$ on $X \times P^{1}$. Of course, in order to talk about the Hermitian-Einstein equation on $X \times P^{1}$ one needs to choose a Kähler metric. We shall consider the one-parameter family of $S U(2)$-invariant Kähler metrics with Kähler form

$$
\omega_{\sigma}=\frac{\sigma}{2} p^{*} \omega_{X} \oplus \omega_{P^{1}}
$$

where $\omega_{P^{1}}$ is the Fubini-Study Kähler form normalized to volume one, and $\sigma \in R^{+}$.

Proposition 2.14 Let $T=\left(E_{1}, E_{2}, \Phi\right)$ be a holomorphic triple and $F$ be the $S U(2)$ equivariant holomorphic bundle over $X \times P^{1}$ associated to $T$, that is given as an extension

$$
0 \longrightarrow p^{*} E_{1} \longrightarrow F \longrightarrow p^{*} E_{2} \otimes q^{*} \mathcal{O}(2) \longrightarrow 0 .
$$

Suppose that $\tau$ and $\tau^{\prime}$ are related by (11) and let

$$
\sigma=\frac{\left(r_{1}+r_{2}\right) \tau-\left(\operatorname{deg} E_{1}+\operatorname{deg} E_{2}\right)}{r_{2}} .
$$

Then $E_{1}$ and $E_{2}$ admit metrics satisfying the coupled $\tau$-vortex equations if and only if $F$ admits an $S U(2)$-invariant Hermitian-Einstein metric with respect to $\omega_{\sigma}$.

Proof. We shall give here just a sketch of the proof (see [GP3, Proposition 3.11] for details). First one has the following result, which is a special case of the general characterization of an $S U(2)$-invariant Hermitian metric on an $S U(2)$-equivariant vector bundle over $X \times P^{1}$.

Lemma 2.15 Let $h$ be an $S U(2)$-invariant Hermitian metric on the bundle $F \longrightarrow$ $X \times P^{1}$ associated to the triple $\left(E_{1}, E_{2}, \Phi\right)$. Then $h$ is of the form

$$
h=p^{*} h_{1} \oplus p^{*} h_{2} \otimes q^{*} h_{2}^{\prime},
$$


where $h_{1}$ and $h_{2}$ are metrics on $E_{1}$ and $E_{2}$, respectively, and $h_{2}^{\prime}$ is an $S U(2)$-invariant metric on $\mathcal{O}(2)$. Conversely, given metrics $h_{1}, h_{2}$ and $h_{2}^{\prime}$ as above, (14) defines an $S U(2)$-invariant metric on $F$.

Let $F_{1}$ and $F_{2}$ be the curvatures of the metric connections of $p^{*} h_{1}$ and $p^{*} h_{2} \otimes q^{*} h_{2}^{\prime}$ respectively. Then

$$
\begin{aligned}
& F_{1}=p^{*} F_{h_{1}} \\
& F_{2}=p^{*} F_{h_{2}} \otimes 1+I_{E_{2}} \otimes q^{*} F_{h_{2}^{\prime}} .
\end{aligned}
$$

The curvature of the metric connection corresponding to $h$ is given by

$$
F_{h}=\left(\begin{array}{cc}
F_{1}-\beta \wedge \beta^{*} & D^{\prime} \beta \\
-D^{\prime \prime} \beta^{*} & F_{2}-\beta^{*} \wedge \beta
\end{array}\right)
$$

where $\beta \in \Omega^{0,1}\left(X \times P^{1}, p^{*}\left(E_{1} \otimes E_{2}^{*}\right) \otimes q^{*} \mathcal{O}(-2)\right)$ is a representative of the extension class in $H^{1}\left(X \times P^{1}, p^{*}\left(E_{1} \otimes E_{2}^{*}\right) \otimes q^{*} \mathcal{O}(-2)\right)$ defining (12), and

$$
D: \Omega^{1}\left(X \times P^{1}, p^{*}\left(E_{1} \otimes E_{2}^{*}\right) \otimes q^{*} \mathcal{O}(-2)\right) \longrightarrow \Omega^{2}\left(X \times P^{1}, p^{*}\left(E_{1} \otimes E_{2}^{*}\right) \otimes q^{*} \mathcal{O}(-2)\right)
$$

is built from the metric connections of $p^{*} h_{1}$ and $p^{*} h_{2} \otimes q^{*} h_{2}^{\prime}$.

As explained in Proposition 2.4, $\beta=p^{*} \Phi \otimes q^{*} \alpha$, where $\alpha \in \Omega^{0,1}\left(P^{1}, \mathcal{O}(-2)\right)$ is the unique $S U(2)$-invariant representative of the element in $H^{1}\left(P^{1}, \mathcal{O}(-2)\right)$, which has to be fixed in order to associate the extension (12) to $\left(E_{1}, E_{2}, \Phi\right)$. One can choose the constant in $H^{1}\left(P^{1}, \mathcal{O}(-2)\right) \cong C$ such that $\alpha \wedge \alpha^{*}=\frac{1}{\sigma} \omega_{P^{1}}$.

Let $\Lambda_{\sigma}$ be the contraction with the Kähler form $\omega_{\sigma}$. A straightforward computation shows that if $\sigma$ is related to $\tau$ by (13), then $h$ is Hermitian-Einstein with respect to $\omega_{\sigma}$. That is

$$
\sqrt{-1} \Lambda_{\sigma} F_{h}=\lambda I_{F}
$$

if and only if $h_{1}$ and $h_{2}$ satisfy the coupled $\tau$-vortex equations.

We have assumed that if the relation between $\sigma$ and $\tau$ is given by (13), then $\sigma>0$. However, we will show in Section 3 that this can actually be derived from the coupled vortex equations.

Remark. The choice of the Kähler metric on $X \times P^{1}$ that we have made differs from the one made in GP3. There the parameter $\sigma$ is multiplying the metric on $P^{1}$, i.e. $\omega_{\sigma}=p^{*} \omega_{X} \oplus \sigma q^{*} \omega_{P^{1}}$. This, and the fact that the volume of $X$ was not normalized to one, explains why the relation between $\tau$ and $\sigma$ given there is the inverse of (13).

\subsection{Invariant Stability and the Hitchin-Kobayashi Correspon- dence}

It is very well-known that the existence of a Hermitian-Einstein metric on a holomorphic vector bundle is governed by the algebraic-geometric condition of stability. Recall 
that a holomorphic vector bundle $E$ over a compact Kähler manifold $(M, \omega)$ is said to be stable if

$$
\mu\left(E^{\prime}\right)<\mu(E)
$$

for every non-trivial coherent subsheaf $E^{\prime} \subset E$. Where

$$
\mu\left(E^{\prime}\right)=\frac{\operatorname{deg} E^{\prime}}{\operatorname{rank} E^{\prime}}
$$

is the slope of $E^{\prime}$.

The precise relation between the Hermitian-Einstein condition and stability is given by the so-called Hitchin-Kobayashi correspondence, proved by Donaldson [D1, D2 in the algebraic case and by Uhlenbeck and Yau [U-Y] for an arbitrary compact Kähler manifold (see also $[\mathrm{Ko}, \mathrm{L}, \mathrm{A-B}, \overline{\mathrm{N}-\mathrm{S}}]$ ):

Theorem 2.16 Let $E$ be a holomorphic vector bundle over a compact Kähler manifold $(M, \omega)$. Then $E$ admits a Hermitian-Einstein metric if and only if $E$ is polystable, that is a direct sum of stable bundles of the same slope.

From this theorem and Proposition 2.14 we conclude that the existence of solutions to the coupled vortex equations must be dictated by the stability of the bundle $F \longrightarrow X \times P^{1}$ associated to the triple $\left(E_{1}, E_{2}, \Phi\right)$. In fact, since the HermitianEinstein metric on $F$ is $S U(2)$-invariant, the condition that $F$ has to satisfy is a slightly weaker condition than stability, namely that of invariant stability. Let $(M, \omega)$ be a compact Kähler manifold and $G$ be a compact Lie group acting on $M$ by isometric biholomorphisms. Let $E$ be a $G$-equivariant holomorphic vector bundle over $M$. We say that $E$ is $G$-invariantly stable if

$$
\mu\left(E^{\prime}\right)<\mu(E)
$$

for every $G$-invariant non-trivial coherent subsheaf $E^{\prime} \subset E$.

The basic relation between $G$-invariant stability and ordinary stability is given by the following theorem (cf. GP2, Theorem 4]).

Theorem 2.17 Let $E$ be a G-invariant holomorphic vector bundle as above. Then $E$ is $G$-invariantly stable if and only if $E$ is $G$-indecomposable and is of the form

$$
E=\bigoplus_{i=1}^{n} E_{i}
$$

where $E_{i}$ is a stable bundle, which is the transformed of $E_{1}$ by an element of $G$.

As a corollary of Theorems 2.16 and 2.17 one obtains a $G$-invariant version of the Hitchin-Kobayashi correspondence (cf. GP2, Theorems 4 and 5]): 
Theorem 2.18 Let $E$ be a G-equivariant holomorphic vector bundle over a compact Kähler manifold $(M, \omega)$. Then $E$ admits a G-invariant Hermitian-Einstein metric if and only if $E$ is $G$-invariantly polystable, that is a direct sum of $G$-invariantly stable bundles of the same slope.

From Proposition 2.14 and Theorem 2.18 we obtain the following existence theorem.

Theorem 2.19 Let $T=\left(E_{1}, E_{2}, \Phi\right)$ be a holomorphic triple over a compact Riemann surface $X$ equipped with a metric. Let $F \longrightarrow X \times P^{1}$ be the bundle associated to $T$ as above. Let $\sigma$ and $\tau$ be real parameters related by (13). Then $E_{1}$ and $E_{2}$ admit metrics satisfying the coupled $\tau$-vortex equations if and only if $F$ is a $S U(2)$-invariantly polystable bundle with respect to the Kähler form $\omega_{\sigma}$ defined above.

\section{Definition and Properties of Stability for Triples}

The existence theorem 2.19 gives conditions on the extension $F \longrightarrow X \times P^{1}$ for existence of solutions to the coupled vortex equations on $\left(E_{1}, E_{2}, \Phi\right)$. We would like to express these conditions entirely in terms of the data on $\left(E_{1}, E_{2}, \Phi\right)$. Indeed this is one of our primary objectives in this paper. To achieve this, we will need an appropriate notion of stability for a triple. In this section we define such a concept for holomorphic triples, and discuss some properties that follow from the definition.

Keeping our earlier notation, we let $E_{1}$ and $E_{2}$ be holomorphic vector bundles over a Riemann surface $X$. We denote their ranks by $r_{1}$ and $r_{2}$ respectively, and their degrees by $d_{1}$ and $d_{2}$. We let $\Phi: E_{2} \longrightarrow E_{1}$ be a holomorphic bundle homomorphism, i.e. $\Phi \in H^{0}\left(\operatorname{Hom}\left(E_{2}, E_{1}\right)\right)$.

Our definition of stability for the triple $T=\left(E_{1}, E_{2}, \Phi\right)$ has two equivalent formulations. The first has some advantages when considering the relation between stability and the coupled vortex equations, while the second has the virtue that it is in the style of the definition of parabolic stability, and thus looks more familiar. Both definitions involve a real parameter, with the result that there is a 1-parameter family of stability criteria for triples. This is the same phenomenon as is observed in the case of holomorphic pairs. All our results can be of course be stated in terms of either definition, and for the sake of completeness we will give both versions.

Definition 3.1 Let $T^{\prime}=\left(E_{1}^{\prime}, E_{2}^{\prime}\right.$, $\left.\Phi\right)$ be a nontrivial subtriple of $\left(E_{1}, E_{2}\right.$, $\left.\Phi\right)$, with rank $E_{1}^{\prime}=r_{1}^{\prime}$ and rank $E_{2}^{\prime}=r_{2}^{\prime}$. For any real $\tau$ define

$$
\theta_{\tau}\left(T^{\prime}\right)=\left(\mu\left(E_{1}^{\prime} \oplus E_{2}^{\prime}\right)-\tau\right)-\frac{r_{2}^{\prime}}{r_{2}} \frac{r_{1}+r_{2}}{r_{1}^{\prime}+r_{2}^{\prime}}\left(\mu\left(E_{1} \oplus E_{2}\right)-\tau\right) .
$$

The triple $T=\left(E_{1}, E_{2}, \Phi\right)$ is called $\tau$-stable if

$$
\theta_{\tau}\left(T^{\prime}\right)<0
$$


for all nontrivial subtriples $T^{\prime}=\left(E_{1}^{\prime}, E_{2}^{\prime}, \Phi\right)$. The triple is called $\tau$-semistable if for all subtriples

$$
\theta_{\tau}\left(T^{\prime}\right) \leq 0
$$

Definition 3.2 With $\sigma$ a real number, define the $\sigma$-degree and $\sigma$-slope of a subtriple $T^{\prime}=\left(E_{1}^{\prime}, E_{2}^{\prime}, \Phi\right)$ by

$$
\operatorname{deg}_{\sigma}\left(T^{\prime}\right)=\operatorname{deg}\left(E_{1}^{\prime} \oplus E_{2}^{\prime}\right)+r_{2}^{\prime} \sigma
$$

and

$$
\mu_{\sigma}\left(T^{\prime}\right)=\frac{\operatorname{deg}_{\sigma}\left(T^{\prime}\right)}{r_{1}^{\prime}+r_{2}^{\prime}}
$$

The triple $T=\left(E_{1}, E_{2}, \Phi\right)$ is called $\sigma$-stable if for all nontrivial subtriples $T^{\prime}=$ $\left(E_{1}^{\prime}, E_{2}^{\prime}, \Phi\right)$ we have

$$
\mu_{\sigma}\left(T^{\prime}\right)<\mu_{\sigma}(T)
$$

A straightforward computation shows the equivalence of these two definitions.

Proposition 3.3 Fix $\tau$ and $\sigma$ such that

$$
\sigma=\frac{r_{1}+r_{2}}{r_{2}}(\tau-\mu(T))
$$

or equivalently

$$
\tau=\mu_{\sigma}(T) .
$$

Then for any subtriple $T^{\prime}=\left(E_{1}^{\prime}, E_{2}^{\prime}, \Phi\right)$, the following are equivalent:

(1) $\theta_{\tau}\left(T^{\prime}\right)<0$

(2) $\mu_{\sigma}\left(T^{\prime}\right)<\mu_{\sigma}(T)$.

That is, the triple is $\tau$-stable if and only if it is $\sigma$-stable. A similar result holds with " $<$ replaced by " = ".

Remark. There are two special cases where the notion of stability for a triple is especially simple, namely when $\Phi=0$, and when $E_{2}$ is a line bundle.

Lemma 3.4 Suppose that $\Phi=0$. The degenerate holomorphic triple $\left(E_{1}, E_{2}, 0\right)$ is $\tau$ semistable if and only if $\tau=\mu\left(E_{1}\right)$ and both bundles are semistable. Such triple cannot be $\tau$-stable.

Proof. Subtriples of $T=\left(E_{1}, E_{2}, 0\right)$ are all of the form $T^{\prime}=\left(E_{1}^{\prime}, E_{2}^{\prime}, 0\right)$, with $E_{1}^{\prime}$ and $E_{2}^{\prime}$ being any holomorphic subbundles of $E_{1}$ and $E_{2}$ respectively. Applying the condition $\theta_{\tau}\left(T^{\prime}\right) \leq 0$ to subtriples of the form $T^{\prime}=\left(E_{1}^{\prime}, 0,0\right)$ gives

$$
\mu\left(E_{1}^{\prime}\right) \leq \tau,
$$


while applying the condition to subtriples of the form $T^{\prime}=\left(E_{1}^{\prime}, E_{2}, 0\right)$ gives stability

$$
\mu\left(E_{1} / E_{1}^{\prime}\right) \geq \tau
$$

These two inequalities imply

$$
\mu\left(E_{1}\right) \leq \tau \leq \mu\left(E_{1}\right)
$$

That is, $\tau=\mu\left(E_{1}\right)$, and hence $E_{1}$ is a semistable bundle. Similarly, by considering the subtriples $\left(0, E_{2}^{\prime}, 0\right)$ and $\left(E_{1}, E_{2}^{\prime}, 0\right)$, we see that $E_{2}$ is also semistable. Notice that the inequalities in (16) and (17) cannot be made strict without leading to a contradiction in $(18)$.

Corollary 3.5 The map $\Phi$ cannot be identically zero in a $\tau$-stable triple.

Lemma 3.6 In the case where $E_{2}=L$ is a line bundle, i.e. $r_{2}=1$, the above definition is equivalent to the notion of $\tau$-stability defined in GP3. It thus corresponds to the $(\tau-\operatorname{deg} L)$-stability for the holomorphic pair $\left(E_{1} \otimes L^{*}, \Phi\right)$.

Proof. In this case there are only two types of subtriple possible, corresponding to $r_{2}^{\prime}=0$ or $r_{2}^{\prime}=1$. In the first case the subtriples are of the form $\left(E_{1}^{\prime}, 0,0\right)$, where $E_{1}^{\prime}$ is an arbitrary holomorphic subbundle of $E_{1}$. The condition $\theta_{\tau}\left(T^{\prime}\right)<0$ then reduces to

$$
\mu\left(E_{1}^{\prime}\right)<\tau
$$

In the second case, the subtriples are of the form $\left(E_{1}^{\prime}, E_{2}, \Phi\right)$ where $E_{1}^{\prime}$ is a holomorphic subbundle such that $\Phi\left(E_{2}\right) \subset E_{1}^{\prime}$. For such subtriples the condition $\theta_{\tau}\left(T^{\prime}\right)<0$ is equivalent to

$$
\left(r_{1}^{\prime}+1\right) \mu\left(E_{1}^{\prime} \oplus E_{2}\right)-\left(r_{1}+1\right) \mu\left(E_{1} \oplus E_{2}\right)-\left(r_{1}^{\prime}-r_{1}\right) \tau<0,
$$

i.e.

$$
\mu\left(E_{1} / E_{1}^{\prime}\right)>\tau
$$

Definition 3.1 can thus be considered a natural extension of the $\tau$-stability for pairs defined in [B2]. For the more general triples which we are considering here however, the number of different possibilities for subtriples is too large to reformulate the definition of $\tau$-stability in the style of GP3 or B2, i.e. in terms of separate slope conditions on the various families of subtriples. The $\tau$-stability of a triple does however imply the following conditions on subtriples:

Proposition 3.7 Let $\left(E_{1}, E_{2}, \Phi\right)$ be a $\tau$-stable triple. Let $\tau^{\prime}$ be related to $\tau$ by

$$
r_{1} \tau+r_{2} \tau^{\prime}=\operatorname{deg} E_{1}+\operatorname{deg} E_{2}
$$

Then 
(1) $\mu\left(E_{1}^{\prime}\right)<\tau$ for all holomorphic subbundles $E_{1}^{\prime} \subset E_{1}$,

(2) $\mu\left(E_{2}^{\prime}\right)<\tau^{\prime}$ for all holomorphic subbundles $E_{2}^{\prime} \subset E_{2}$ such that $E_{2}^{\prime} \subset \operatorname{Ker}(\Phi)$,

(3) $\mu\left(E_{2}^{\prime \prime}\right)>\tau^{\prime}$ for all holomorphic quotients of $E_{2}$,

(4) $\mu\left(E_{1}^{\prime \prime}\right)>\tau$ for all holomorphic quotients of $E_{1}$ such that $\pi \circ \Phi\left(E_{2}\right)=0$, where $\pi: E_{1} \longrightarrow E_{1}^{\prime \prime}$ denotes projection onto the quotient.

Proof. These are immediate consequences of the stability condition, i.e.

$$
\theta_{\tau}\left(E_{1}^{\prime}, E_{2}^{\prime}, \Phi^{\prime}\right)<0,
$$

applied to the following special subtriples

(1) $\left(E_{1}^{\prime}, 0, \Phi\right)$,

(2) $\left(0, E_{2}^{\prime}, \Phi\right)$,

(3) $\left(E_{1}, E_{2}^{\prime}, \Phi\right)$, with $E_{2}^{\prime \prime}=E_{2} / E_{2}^{\prime}$,

(4) $\left(E_{1}^{\prime}, E_{2}, \Phi\right)$, with $E_{1}^{\prime \prime}=E_{1} / E_{1}^{\prime}$.

Notice that (19) can be expressed as

$$
\tau^{\prime}=\mu\left(E_{1} \oplus E_{2}\right)-\frac{r_{1}}{r_{1}+r_{2}} \sigma .
$$

An equivalent formulation of Proposition 3.7 is thus

Proposition 3.8 Let $T=\left(E_{1}, E_{2}, \Phi\right)$ be a $\sigma$-stable triple. Then

(1) $\mu\left(E_{1}^{\prime}\right)<\mu(T)+\frac{r_{2}}{r_{1}+r_{2}} \sigma$ for all holomorphic subbundles $E_{1}^{\prime} \subset E_{1}$,

(2) $\mu\left(E_{2}^{\prime}\right)<\mu(T)-\frac{r_{1}}{r_{1}+r_{2}} \sigma$ for all holomorphic subbundles $E_{2}^{\prime} \subset E_{2}$ such that $E_{2}^{\prime} \subset \operatorname{Ker} \Phi$

(3) $\mu\left(E_{2}^{\prime \prime}\right)>\mu(T)-\frac{r_{1}}{r_{1}+r_{2}} \sigma$ for all holomorphic quotients, $E_{2}^{\prime \prime}$, of $E_{2}$,

(4) $\mu\left(E_{1}^{\prime \prime}\right)>\mu(T)+\frac{r_{2}}{r_{1}+r_{2}} \sigma$ for all holomorphic quotients, $E_{1}^{\prime \prime}$, of $E_{1}$ such that $\pi \circ \Phi\left(E_{2}\right)=0$, where $\pi: E_{1} \longrightarrow E_{1}^{\prime \prime}$ denotes projection onto the quotient.

\subsection{Stable implies simple}

An important consequence of stability for holomorphic bundles is that the only automorphisms of a stable bundle are the constant multiples of the identity, i.e. stable bundles are simple. We now show that this remains true in the case of holomorphic triples, where the definition of simplicity is that given in Definition 2.8. The key result is the following Proposition.

Proposition 3.9 Let $\left(E_{1}, E_{2}, \Phi\right)$ be a $\tau$-stable holomorphic triple. Let $(u, v)$ be in $H^{0}\left(E_{1}, E_{2}, \Phi\right)$. Either $(u, v)$ is trivial, or both $u$ and $v$ are isomorphisms. 
Proof. Suppose that $u$ and $v$ are both neither trivial nor isomorphisms. Consider the triples $K=(\operatorname{Ker} u, \operatorname{Ker} v, \Phi)$ and $I=(\operatorname{Im} u, \operatorname{Im} v, \Phi)$, where Ker and $\operatorname{Im}$ denotes the kernels and images of the maps. Since $u \Phi=\Phi v$, these are both proper subtriples of $\left(E_{1}, E_{2}, \Phi\right)$, and thus the $\tau$-stability condition gives

$$
\theta_{\tau}(K)<0
$$

and

$$
\theta_{\tau}(I)<0 \text {. }
$$

We also have the exact sequences

$$
0 \longrightarrow \operatorname{Ker} u \longrightarrow E_{1} \longrightarrow \operatorname{Im} u \longrightarrow 0
$$

and

$$
0 \longrightarrow \operatorname{Ker} v \longrightarrow E_{2} \longrightarrow \operatorname{Im} v \longrightarrow 0 .
$$

Let $\sigma_{u}$ and $\rho_{u}$ denote the ranks of $\operatorname{Ker} u$ and $\operatorname{Im} u$, and similarly for $\sigma_{v}$ and $\rho_{v}$. Then from the exact sequences we get

$$
\left(\sigma_{u}+\sigma_{v}\right) \mu(\operatorname{Ker} u \oplus \operatorname{Ker} v)+\left(\rho_{u}+\rho_{v}\right) \mu(\operatorname{Im} u \oplus \operatorname{Im} v)=\left(r_{1}+r_{2}\right) \mu\left(E_{1} \oplus E_{2}\right) .
$$

But by definition of $\theta_{\tau}(K)$,

$$
\begin{aligned}
\left(\sigma_{u}+\sigma_{v}\right) \mu(\operatorname{Ker} u \oplus \operatorname{Ker} v) & =\left(\sigma_{u}+\sigma_{v}\right) \theta_{\tau}(K) \\
& +\frac{\sigma_{v}}{r_{2}}\left(r_{1}+r_{2}\right) \mu\left(E_{1} \oplus E_{2}\right)+\left(\sigma_{u}+\sigma_{v}-\frac{\sigma_{v}}{r_{2}}\left(r_{1}+r_{2}\right)\right) \tau,
\end{aligned}
$$

with a similar expression for $\left(\rho_{u}+\rho_{v}\right)\left(\mu(\operatorname{Im} u \oplus \operatorname{Im} v)\right.$. Also, $\sigma_{u}+\rho_{u}=r_{1}$, and $\sigma_{v}+\rho_{v}=$ $r_{2}$. Hence from (22) we obtain

$$
r_{1} \theta_{\tau}(K)+r_{2} \theta_{\tau}(I)=0 .
$$

This is incompatible with (20) and (21).

Corollary 3.10 If $\left(E_{1}, E_{2}, \Phi\right)$ is $\tau$-stable, then it is simple.

Proof. Let $(u, v)$ be a nontrivial element in $H^{0}\left(E_{1}, E_{2}, \Phi\right)$. By the above Proposition, both $u$ and $v$ are isomorphisms. Fix a point $p$ on the base of the bundles, and let $\lambda$ be an eigenvalue of $v:\left.\left.E_{2}\right|_{p} \longrightarrow E_{2}\right|_{p}$, i.e. of $v$ acting on the fibre over $p$.

Now define

$$
\begin{aligned}
& \hat{u}=u-\lambda I_{1}, \\
& \hat{v}=v-\lambda I_{2} .
\end{aligned}
$$

Clearly $(\hat{u}, \hat{v})$ is in $H^{0}\left(E_{1}, E_{2}, \Phi\right)$, but since $\hat{u}$ is not an isomorphism, it follows from Proposition 3.9 that both are identically zero, i.e.

$$
(u, v)=\lambda\left(I_{1}, I_{2}\right)
$$


We see, in particular, that stable triples are necessarily irreducible. For reducible triples, we can however define a notion of polystability. This will be useful when we consider the relation between stability and the coupled vortex equations.

Definition 3.11 Let $T=\left(E_{1}, E_{2}, \Phi\right)$ be a reducible triple, with $T=\bigoplus_{i=1}^{n} T_{i}$. Suppose that in each summand $T_{i}=\left(E_{1 i}, E_{2 i}, \Phi_{i}\right)$, the map $\Phi_{i}$ is non-trivial unless $E_{1 i}=0$ or $E_{2 i}=0$. Fix value of $\tau$, and let $\tau^{\prime}$ be related to $\tau$ as in (19). We say that $T$ is $\tau$-polystable if for each summand $T_{i}$

(1) if $\Phi_{i} \neq 0$, then $T_{i}$ is $\tau$-stable,

(2) if $E_{1 i}=0$, then $E_{2 i}$ is a stable bundle of slope $\tau^{\prime}$,

(3) if $E_{2 i}=0$, then $E_{1 i}$ is a stable bundle of slope $\tau$.

\subsection{Duality for triples}

Associated to a triple $T=\left(E_{1}, E_{2}, \Phi\right)$ there is always a dual triple $T^{*}=\left(E_{2}^{*}, E_{1}^{*}, \Phi^{*}\right)$, where $\Phi^{*}$ is the transpose of $\Phi$, i.e. the image of $\Phi$ via the canonical isomorphism

$$
\operatorname{Hom}\left(E_{2}, E_{1}\right) \cong \operatorname{Hom}\left(E_{1}^{*}, E_{2}^{*}\right)
$$

It is reasonable that the stability of $T$ should be related to that of $T^{*}$. More precisely.

Proposition $3.12 T=\left(E_{1}, E_{2}, \Phi\right)$ is $\tau$-stable if and only if $T^{*}=\left(E_{2}^{*}, E_{1}^{*}, \Phi^{*}\right)$ is $\left(-\tau^{\prime}\right)$-stable, where $\tau^{\prime}$ is related to $\tau$ by (19). Equivalently, $T$ is $\sigma$-stable if and only if $T^{*}$ is $\sigma$-stable.

Proof. Let $T^{\prime}=\left(E_{1}^{\prime}, E_{2}^{\prime}, \Phi^{\prime}\right)$ be a subtriple of $T$. This defines a quotient triple $T^{\prime \prime}=$ $\left(E_{1}^{\prime \prime}, E_{2}^{\prime \prime}, \Phi^{\prime \prime}\right)$, where $E_{1}^{\prime \prime}=E_{1} / E_{1}^{\prime}, E_{2}^{\prime \prime}=E_{2} / E_{2}^{\prime}$, and $\Phi^{\prime \prime}$ is the morphism induced by $\Phi . T^{\prime \prime *}=\left(E_{2}^{\prime \prime *}, E_{1}^{\prime \prime *}, \Phi^{\prime \prime *}\right)$ is the desired subtriple of $T^{*}$. Since one has the isomorphism $T \cong T^{* *}$ we can conclude that there is a one-to-one correspondence between subtriples of $T$ and subtriples of $T^{*}$. It is not difficult to verify that $\theta_{\tau}\left(T^{\prime}\right)<0$ is equivalent to $\theta_{-\tau^{\prime}}\left(T^{\prime \prime *}\right)<0$. The equivalence of the $\sigma$-stability for $T$ and $T^{*}$ now follows from the fact that if $\tau=\mu_{\sigma}(T)$, then

$$
-\tau^{\prime}=-\mu\left(E_{1} \oplus E_{2}\right)-\frac{r_{1}}{r_{1}+r_{2}} \sigma=-\mu_{\sigma}\left(T^{*}\right) .
$$

\subsection{Constraints on the parameters}

Proposition 3.13 Let $\left(E_{1}, E_{2}, \Phi\right)$ be a $\tau$-stable triple, and let $\tau^{\prime}$ be as above. Then 
(1) $\tau>\mu\left(E_{1}\right)$,

(2) $\tau^{\prime}<\mu\left(E_{2}\right)$, and

(3) $\tau-\tau^{\prime}>0$,

Equivalently, if $\left(E_{1}, E_{2}, \Phi\right)$ is $\sigma$-stable, then

(1) $\sigma>\mu\left(E_{1}\right)-\mu\left(E_{2}\right)$,

(2) $\sigma>0$.

Proof. The first two statement follows from cases (1) and (3) in Proposition 3.7 with $E_{1}^{\prime}=E_{1}$, and $E_{2}^{\prime \prime}=E_{2}$ respectively.

To prove the third statement, let $K$ be the subbundle of $E_{2}$ generated by the kernel of $\Phi$, and let $I$ be the subbundle of $E_{1}$ generated by the image of $\Phi$. Since the triple is assumed to be $\tau$-stable, $\Phi$, and therefore $I$, is non-trivial. By (1) in Proposition 3.7 we thus have

$$
\mu(I)<\tau .
$$

But we also have $0 \longrightarrow K \longrightarrow E_{2} \longrightarrow I \longrightarrow 0$, i.e. $I$ is a quotient of $E_{2}$. It thus follows from (3) in Proposition 3.7 that

$$
\mu(I)>\tau^{\prime}
$$

The bounds on $\sigma$ can be obtained from those on $\tau$ by substituting $\tau=\mu_{\sigma}(T)$, and using the fact that

$$
\sigma=\tau-\tau^{\prime}
$$

if $\tau^{\prime}$ is as above.

Part (1) of this proposition gives the lower bound on the allowed range for $\tau$. In almost all cases the rank and degree of $E_{1}$ and $E_{2}$ also impose an upper bound on $\tau$. In fact

Proposition 3.14 Let $\left(E_{1}, E_{2}, \Phi\right)$ be a triple with $r_{1} \neq r_{2}$. If the triple is $\tau$-stable then

$$
\tau<\mu\left(E_{1}\right)+\frac{r_{2}}{\left|r_{1}-r_{2}\right|}\left(\mu\left(E_{1}\right)-\mu\left(E_{2}\right)\right)
$$

Equivalently, if the triple is $\sigma$-stable, then

$$
\sigma<\left(1+\frac{r_{1}+r_{2}}{\left|r_{1}-r_{2}\right|}\right)\left(\mu\left(E_{1}\right)-\mu\left(E_{2}\right)\right) .
$$

Proof. Let $K=\operatorname{Ker} \Phi$ and $I=\operatorname{Im} \Phi$. Consider the subtriples

$$
T_{1}=(0, K, \Phi) \quad \text { and } \quad T_{2}=\left(I, E_{2}, \Phi\right)
$$

Since $r_{1} \neq r_{2}, \Phi$ cannot be an isomorphism and at least one of these must be a proper subtriple. Let $r_{2}^{\prime}=\operatorname{rank} K, r_{2}^{\prime \prime}=\operatorname{rank} I, d_{2}^{\prime}=\operatorname{deg} K$ and $d_{2}^{\prime \prime}=\operatorname{deg} I$. 
A straightforward computation shows that

$$
\begin{aligned}
& \theta_{\tau}\left(T_{1}\right)<0 \Longleftrightarrow d_{2}^{\prime}-r_{2}^{\prime}\left(d_{1}+d_{2}\right)+r_{1} r_{2}^{\prime} \tau<0 \\
& \theta_{\tau}\left(T_{2}\right)<0 \Longleftrightarrow d_{2}^{\prime \prime}-d_{1}+\left(r_{1}-r_{2}^{\prime \prime}\right) \tau<0 .
\end{aligned}
$$

Adding (27) to $r_{2}$ times (28), and noting that $d_{2}=d_{2}^{\prime}+d_{2}^{\prime \prime}$ and $r_{2}=r_{2}^{\prime}+r_{2}^{\prime \prime}$, we get that

$$
r_{2}\left(d_{2}-d_{1}\right)-r_{2}^{\prime}\left(d_{1}+d_{2}\right)+\left(r_{2}\left(r_{1}-r_{2}\right)+r_{2}^{\prime}\left(r_{1}+r_{2}\right)\right) \tau<0 .
$$

On the other hand combining (3) in Proposition 3.13 and (19) we obtain

$$
d_{1}+d_{2}-\left(r_{1}+r_{2}\right) \tau<0
$$

Adding (29) to $r_{2}^{\prime}$ times (30) we get

$$
\left(r_{1}-r_{2}\right) \tau<d_{1}-d_{2}
$$

If now $r_{1}>r_{2}$, then we get

$$
\tau<\frac{d_{1}-d_{2}}{r_{1}-r_{2}}
$$

or equivalently

$$
\tau<\mu\left(E_{1}\right)+\frac{r_{2}}{r_{1}-r_{2}}\left(\mu\left(E_{1}\right)-\mu\left(E_{2}\right)\right)
$$

To obtain the bound in the case $r_{1}<r_{2}$, note that by Proposition 3.12 the $\tau$-stability of $\left(E_{1}, E_{2}, \Phi\right)$ is equivalent to the $\left(-\tau^{\prime}\right)$-stability of the dual triple $\left(E_{2}^{*}, E_{1}^{*}, \Phi^{*}\right)$, where $\tau^{\prime}$ is given by

$$
r_{1} \tau+r_{2} \tau^{\prime}=d_{1}+d_{2}
$$

Hence we can apply (31) to $\left(E_{2}^{*}, E_{1}^{*}, \Phi^{*}\right)$ to get that

$$
-\tau^{\prime}<\frac{d_{1}-d_{2}}{r_{2}-r_{1}}
$$

which together with (32) leads to

$$
\tau<\frac{\left(2 r_{2}-r_{1}\right) d_{1}-r_{1} d_{2}}{\left(r_{2}-r_{1}\right) r_{1}}
$$

i.e.

$$
\tau<\mu\left(E_{1}\right)+\frac{r_{2}}{r_{2}-r_{1}}\left(\mu\left(E_{1}\right)-\mu\left(E_{2}\right)\right)
$$

Combining the lower and upper bounds on $\tau$ (or $\sigma$ ) we can deduce

Corollary 3.15 If rank $E_{1}$ and rank $E_{2}$ are unequal, then a triple $\left(E_{1}, E_{2}, \Phi\right)$ cannot be stable unless $\mu\left(E_{2}\right)<\mu\left(E_{1}\right)$. 
Furthermore, by the proof of Proposition 3.14 we get the following corollary.

Corollary 3.16 Let $\left(E_{1}, E_{2}, \Phi\right)$ be $\tau$-stable and suppose that $r=s$. If $\Phi$ is not an isomorphism, then $d_{1}>d_{2}$. In particular, in any $\tau$-stable triple $\left(E_{1}, E_{2}, \Phi\right)$, the bundle map $\Phi$ is an isomorphism if and only if $r_{1}=r_{2}$ and $d_{1}=d_{2}$.

Proof. The fact that $d_{1}>d_{2}$ follows from the inequality

$$
\left(r_{1}-r_{2}\right) \tau<d_{1}-d_{2}
$$

which applies if $\Phi$ is not an isomorphism. In particular, if $\Phi$ is not an isomorphism then $d_{1} \neq d_{2}$. Conversely, if $\Phi$ is an isomorphism, then clearly $r_{1}=r_{2}$ and $d_{1}=d_{2}$.

It is when $\Phi$ is an isomorphism that the range for $\tau$ can fail to be bounded. For example

Proposition 3.17 Suppose that $E_{1}$ and $E_{2}$ are both stable bundles of rank $r$ and degree $d$, and that $\Phi: E_{2} \longrightarrow E_{1}$ is non trivial. Then for any $\tau>\mu\left(E_{1}\right)$ the holomorphic triple $\left(E_{1}, E_{2}, \Phi\right)$ is $\tau$-stable.

Proof. Let $\mu(T)=\mu\left(E_{1} \oplus E_{2}\right)$, and for a subtriple $T^{\prime}=\left(E_{1}^{\prime}, E_{2}^{\prime}, \Phi\right)$ set $\mu\left(T^{\prime}\right)=$ $\mu\left(E_{1}^{\prime} \oplus E_{2}^{\prime}\right)$. Since $E_{1}$ and $E_{2}$ are stable and of equal slope, we have $\mu\left(T^{\prime}\right)<\mu(T)$ for all subtriples. Thus

$$
\theta_{\tau}\left(T^{\prime}\right) \leq(\mu(T)-\tau) \frac{r_{1}^{\prime}-r_{2}^{\prime}}{r_{1}^{\prime}+r_{2}^{\prime}}
$$

Since $\Phi$ is a nontrivial map between stable bundles of the same rank and degree, it must be a multiple of the identity (cf. O-S-S ). In particular $\Phi$ is injective and hence $r_{1}^{\prime}-r_{2}^{\prime} \geq 0$. Thus $\theta_{\tau}\left(T^{\prime}\right) \leq 0$. In fact, $\theta_{\tau}\left(T^{\prime}\right)<0$ unless $r_{1}^{\prime}=r_{2}^{\prime}$. But in that case, we can write

$$
\theta_{\tau}\left(T^{\prime}\right)=r_{1}^{\prime}\left(\mu\left(E_{1}^{\prime}\right)-\mu\left(E_{1}\right)\right)+r_{1}^{\prime}\left(\mu\left(E_{2}^{\prime}\right)-\mu\left(E_{2}\right)\right),
$$

which is strictly negative.

\subsection{Special values for $\tau$ and $\tau$-semistability}

In principle $\tau$ is a continuously varying real parameter. The stability properties of a given triple do not likewise vary continuously, but can change only at certain rational values of $\tau$. This is the same phenomenon as appears in the case of stable pairs. In both cases it is due to the fact that, except for $\tau$ itself, all numerical quantities in the definition of stability are rational numbers with bounded denominators. In the case of holomorphic pairs, this has the additional consequence that for the generic choice of $\tau$ there is no distinction between stability and semistablity. This is in contrast to the case of pure bundles, where the notions of stability and semistability coincide only when the rank and degree of the bundle are coprime. The next proposition shows that for a holomorphic triple both the value of $\tau$ and the greatest common divisor of the rank and degree, are relevant. 
Proposition 3.18 Let $T=\left(E_{1}, E_{2}, \Phi\right)$ be a $\tau$-semistable triple, and let $T^{\prime}=\left(E_{1}^{\prime}, E_{2}^{\prime}, \Phi^{\prime}\right)$ be a subtriple such that $\theta_{\tau}\left(T^{\prime}\right)=0$. Then either

$$
r_{1} r_{2}^{\prime}=r_{2} r_{1}^{\prime} \text { and } \mu\left(E_{1}^{\prime} \oplus E_{2}^{\prime}\right)=\mu\left(E_{1} \oplus E_{2}\right),
$$

or

$$
\frac{r_{2}\left(r_{1}^{\prime}+r_{2}^{\prime}\right) \mu\left(T^{\prime}\right)-r_{2}^{\prime}\left(r_{1}+r_{2}\right) \mu(T)}{r_{2} r_{1}^{\prime}-r_{1} r_{2}^{\prime}}=\tau .
$$

In particular, if $r_{1}+r_{2}$ and $d_{1}+d_{2}$ are coprime, and $\tau$ is a not rational number with denominator of magnitude less than $r_{1} r_{2}$, then all $\tau$-semistable triples are $\tau$-stable.

Proof. From the definition of $\theta_{\tau}$, we see that $\theta_{\tau}\left(T^{\prime}\right)=0$ is equivalent to

$$
\left(\mu\left(E_{1}^{\prime} \oplus E_{2}^{\prime}\right)-\frac{r_{2}^{\prime}}{r_{2}} \frac{r_{1}+r_{2}}{r_{1}^{\prime}+r_{2}^{\prime}} \mu\left(E_{1} \oplus E_{2}\right)\right)=\tau \frac{r_{1}^{\prime} r_{2}-r_{1} r_{2}^{\prime}}{r_{1}^{\prime} r_{2}+r_{2}^{\prime} r_{2}} .
$$

If $r_{1}^{\prime} r_{2}-r_{1} r_{2}^{\prime} \neq 0$ we get (??), and if $r_{1}^{\prime} r_{2}-r_{1} r_{2}^{\prime}=0$ then

$$
\frac{r_{2}^{\prime}}{r_{2}} \frac{r_{1}+r_{2}}{r_{1}^{\prime}+r_{2}^{\prime}}=1
$$

and we get (33).

Next we compare the stability conditions for a triple and for the two bundles in the triple.

Proposition 3.19 Let $\left(E_{1}, E_{2}, \Phi\right)$ be a non-degenerate holomorphic triple. There is an $\epsilon>0$, which depends only on the degrees and ranks of $E_{1}$ and $E_{2}$, and such that for $\mu\left(E_{1}\right)<\tau<\mu\left(E_{1}\right)+\epsilon$ the following is true:

(1) If $\left(E_{1}, E_{2}, \Phi\right)$ is a $\tau$-stable triple, then both $E_{1}$ and $E_{2}$ are semistable bundles.

(2) Conversely, if $E_{1}$ and $E_{2}$ are stable bundles, then $\left(E_{1}, E_{2}, \Phi\right)$ will be a $\tau$-stable triple for any choice of $\Phi \in H^{0}\left(\operatorname{Hom}\left(E_{2}, E_{1}\right)\right)$.

Proof. For all subbundles $E_{1}^{\prime} \subset E_{1}$ the slope $\mu\left(E_{1}^{\prime}\right)$ is a rational number with denominator less than $r_{1}$. Clearly, if we pick $\epsilon$ small enough then the interval $\left(\mu\left(E_{1}\right), \mu\left(E_{1}\right)+\epsilon\right)$ contains no rational numbers with denominator less than $r_{1}$. The condition $\mu\left(E_{1}^{\prime}\right)<\tau$ is thus equivalent to the condition $\mu\left(E_{1}^{\prime}\right) \leq \mu\left(E_{1}\right)$, i.e. to the semistability of $E_{1}$.

Furthermore, as noted above, if $\tau<\mu\left(E_{1}\right)+\epsilon$ then $\tau^{\prime}>\mu\left(E_{2}\right)-\frac{r_{1}}{r_{2}} \epsilon$. Hence if $\frac{r_{1}}{r_{2}} \epsilon$ is small enough, then the condition $\mu\left(E_{2} / E_{2}^{\prime}\right)>\tau^{\prime}$ for all subbundles $E_{2}^{\prime} \subset E_{2}$ becomes equivalent to the condition that $\mu\left(E_{2} / E_{2}^{\prime}\right) \geq \mu\left(E_{2}\right)$.

Conversely, suppose $\tau=\mu\left(E_{1}\right)+\delta$ for some $\delta>0$, and that $\Phi$ is any section of $H^{0}\left(\operatorname{Hom}\left(E_{2}, E_{1}\right)\right)$. Then for any subtriple $\left(E_{1}^{\prime}, E_{2}^{\prime}, \Phi\right)$ we get

$$
\left(r_{1}^{\prime}+r_{2}^{\prime}\right) \theta_{\tau}\left(E_{1}^{\prime}, E_{2}^{\prime}, \Phi^{\prime}\right)=r_{1}^{\prime}\left(\mu\left(E_{1}^{\prime}\right)-\mu\left(E_{1}\right)\right)+r_{2}^{\prime}\left(\mu\left(E_{2}^{\prime}\right)-\mu\left(E_{2}\right)\right)+\left(r_{2} r_{1}^{\prime}-r_{1} r_{2}^{\prime}\right) \delta,
$$

where $r_{1}^{\prime}=\operatorname{rank} E_{1}^{\prime}$ and $r_{2}^{\prime}=\operatorname{rank} E_{2}^{\prime}$. If $E_{1}$ and $E_{2}$ are stable, and $\delta$ is small enough, then it follows from this that $\theta_{\tau}\left(E_{1}^{\prime}, E_{2}^{\prime}, \Phi^{\prime}\right)<0$ for all subtriples. 


\section{Main theorem}

In this section we shall show how the stability of a holomorphic triple over $X$ relates to the stability of the associated ( $\mathrm{SU}(2)$-equivariant) bundle over $X \times P^{1}$. As in Section 2. let $F \longrightarrow X \times P^{1}$ be the extension associated to the triple $\left(E_{1}, E_{2}, \Phi\right)$, i.e. let $F$ be

$$
0 \longrightarrow p^{*} E_{1} \longrightarrow F \longrightarrow p^{*} E_{2} \otimes q^{*} \mathcal{O}(2) \longrightarrow 0,
$$

where $p$ and $q$ are the projections from $X \times P^{1}$ to $X$ and $P^{1}$ respectively, and $\mathcal{O}(2)$ is the line bundle of degree 2 over $P^{1}$. To relate the $\tau$-stability of $\left(E_{1}, E_{2}, \Phi\right)$ to the stability of $F$ we need to consider some Kähler polarization on $X \times P^{1}$. The parameter $\tau$ will be encoded in this polarization. Let us choose a metric on $X$ with Kähler form $\omega_{X}$, with volume normalized to one. The metric we shall consider on $X \times P^{1}$ will be, as in Section 2, the product of a the metric on $X$ with a coefficient depending on a parameter $\sigma>0$, and the Fubini-Study metric on $P^{1}$ with volume also normalized to one. The Kähler form corresponding to this metric depending on the parameter $\sigma$ is

$$
\omega_{\sigma}=\frac{\sigma}{2} p^{*} \omega_{X} \oplus q^{*} \omega_{P^{1}}
$$

We can now state the main result of this section.

Theorem 4.1 Let $\left(E_{1}, E_{2}, \Phi\right)$ be a holomorphic triple over a compact Riemann surface $X$. Let $F$ be the holomorphic bundle over $X \times P^{1}$ defined by $\left(E_{1}, E_{2}, \Phi\right)$ as in Proposition 2.4, and let

$$
\sigma(\tau)=\frac{\left(r_{1}+r_{2}\right) \tau-\left(\operatorname{deg} E_{1}+\operatorname{deg} E_{2}\right)}{r_{2}} .
$$

Suppose that in $\left(E_{1}, E_{2}, \Phi\right)$ the two bundles $E_{1}$ and $E_{2}$ are not isomorphic. Then $\left(E_{1}, E_{2}, \Phi\right)$ is $\tau$-stable (equivalently $\sigma$-stable) if and only if $F$ is stable with respect to $\omega_{\sigma}$.

In the case that $E_{1} \cong E_{2} \cong E$, the triple $(E, E, \Phi)$ is $\tau$-stable (equivalently $\sigma$-stable) if and only if $F$ decomposes as a direct sum

$$
F=p^{*} E \otimes q^{*} \mathcal{O}(1) \oplus p^{*} E \otimes q^{*} \mathcal{O}(1),
$$

and $p^{*} E \otimes q^{*} \mathcal{O}(1)$ is stable with respect to $\omega_{\sigma}$.

Proof. As mentioned in $\oint 3$, if $E_{2}$ is a line bundle the $\tau$-stability of $\left(E_{1}, E_{2}, \Phi\right)$ is equivalent to the $\tau$-stability of the pair $\left(E_{1} \otimes E_{2}^{*}, \Phi\right)$ in the sense of Bradlow [B2]. In this case Theorem 4.1 has been proved in GP3, Theorem 4.6.]. The main ideas of that proof extend to the general case in a rather straightforward manner.

Recall that the bundle $F$ associated to $\left(E_{1}, E_{2}, \Phi\right)$ comes equipped with a holomorphic action of $S U(2)$. It makes sense therefore to talk about the $S U(2)$-invariant stability of $F$. As explained in $\S 2$, this is like ordinary stability, but the slope condition has to be satisfied only for $S U(2)$-invariant subsheaves of $F$. In order to prove the theorem we shall prove first the following slightly weaker result. 
Proposition 4.2 Let $\left(E_{1}, E_{2}, \Phi\right)$ be a holomorphic triple over a compact Riemann surface $X$. Let $F$ be the holomorphic bundle over $X \times P^{1}$ defined by $\left(E_{1}, E_{2}, \Phi\right)$ and let $\sigma$ and $\tau$ be related by (37). Then $\left(E_{1}, E_{2}, \Phi\right)$ is $\tau$-stable (equivalently $\sigma$-stable) if and only if $F$ is $S U(2)$-invariantly stable with respect to $\omega_{\sigma}$.

Proof. We saw in 52 (Lemmas 2.6 and 2.7) that there is a one-to-one correspondence between subtriples $T^{\prime}=\left(E_{1}^{\prime}, E_{2}^{\prime}, \Phi^{\prime}\right)$ of $T$ and $S U(2)$-invariant coherent subsheaves $F^{\prime} \subset F$. Moreover, the subsheaf $F^{\prime}$ defined by $T^{\prime}$ is an extension of the form

$$
0 \longrightarrow p^{*} E_{1}^{\prime} \longrightarrow F^{\prime} \longrightarrow p^{*} E_{2}^{\prime} \otimes q^{*} \mathcal{O}(2) \longrightarrow 0 .
$$

In terms of the parameter $\tau^{\prime}$ as defined in (19), the relation between $\sigma$ and $\tau$, given by (37), can be rewritten as

$$
\sigma=\tau-\tau^{\prime}
$$

If $\left(E_{1}, E_{2}, \Phi\right)$ is $\tau$-stable (equivalently, $\sigma$-stable) it follows from (3) in Proposition 3.13 that $\sigma$ defined by (37) is positive. The slope of $F^{\prime}$ with respect to $\omega_{\sigma}$ is defined as

$$
\mu_{\sigma}\left(F^{\prime}\right)=\frac{\operatorname{deg}_{\sigma} F^{\prime}}{\operatorname{rank} F^{\prime}},
$$

where $\operatorname{deg}_{\sigma} F^{\prime}$ is the degree of $F^{\prime}$, which is given by

$$
\operatorname{deg}_{\sigma} F=\frac{1}{2} \int_{X \times P^{1}} c_{1}(F) \wedge \omega_{\sigma} .
$$

The proposition follows now from the following lemma.

Lemma 4.3 Let $T^{\prime}$ be a subtriple of $T$ and $F^{\prime}$ the corresponding $S U(2)$-invariant subsheaf of $F$. Let $\sigma$ be as in Proposition 4.9 . The following are equivalent

(1) $\mu_{\sigma}\left(F^{\prime}\right)<\mu_{\sigma}(F)$

(2) $\theta_{\tau}\left(T^{\prime}\right)<0$

(3) $\mu_{\sigma}\left(T^{\prime}\right)<\mu_{\sigma}(T)$.

Proof. The equivalence between (2) and (3) corresponds, of course, to the two equivalent definitions of stability for $T$ (cf. Proposition 3.3).

From (35) and (38) we obtain that

$$
\mu_{\sigma}(F)=\frac{\operatorname{deg} E_{1}+\operatorname{deg} E_{2}+\sigma r_{2}}{r_{1}+r_{2}},
$$

and

$$
\mu_{\sigma}\left(F^{\prime}\right)=\frac{\operatorname{deg} E_{1}^{\prime}+\operatorname{deg} E_{2}^{\prime}+\sigma r_{2}^{\prime}}{r_{1}^{\prime}+r_{2}^{\prime}},
$$

where $r_{1}^{\prime}=\operatorname{rank} E_{1}^{\prime}$ and $r_{2}^{\prime}=\operatorname{rank} E_{2}^{\prime}$. From Definition 3.1 we immediately obtain the equivalence between (1) and (3). 
Remark. As usual, in order for $F$ to be $S U(2)$-invariantly stable it is enough to check condition (1) of Lemma 4.3 only for saturated $S U(2)$-invariant subsheaves, that is $S U(2)$-invariant subsheaves $F^{\prime}$ such that the quotient $F / F^{\prime}$ is torsion-free. Such a subsheaf $F^{\prime}$ is a subbundle outside of a set of codimension greater or equal than 2 . Hence by $S U(2)$-invariance one concludes that $F^{\prime}$ must be actually a subbundle of $F$ over the whole $X \times P^{1}$. It is easy to see that the saturation of $F^{\prime}$ implies that of $p^{*} E_{1}^{\prime}$ and $p^{*} E_{2}^{\prime} \otimes q^{*} \mathcal{O}(2)$ in (B8), and hence $E_{1}^{\prime} \subset E_{1}$ and $E_{2}^{\prime} \subset E_{2}$ are in fact subbundles. In other words, the one-to-one correspondence between $S U(2)$-invariant subsheaves of $F$ and subtriples of $T$ established in Lemma 2.7 sends saturated subsheaves into saturated subtriples.

To prove the theorem, we first observe that if $F$ is $S U(2)$-invariantly stable then, from Theorem 2.17, it decomposes as a direct sum

$$
F=F_{1} \oplus F_{2} \oplus \ldots \oplus F_{k}
$$

of stable bundles, where $F_{i}$ is the transformed by an element of $S U(2)$ of a fixed subbundle $F_{1}$ of $F$.

For the remaining parts of the theorem, the proof splits into two cases, corresponding to whether $E_{1}$ and $E_{2}$ are isomorphic (as holomorphic bundles) or not. We treat the non-isomorphic case first. Notice that in this case, the map $\Phi$ certainly cannot be an isomorphism. Clearly if $F$ is stable it is in particular $S U(2)$-invariantly stable and hence by the previous Proposition, the corresponding triple will be $\tau$-stable. Suppose now that $\left(E_{1}, E_{2}, \Phi\right)$ is $\tau$-stable, and that $\Phi$ is not an isomorphism. Our strategy to prove the stability of $F$ will be to prove that $F$ is simple, that is $H^{0}$ (End $\left.F\right) \cong C$, and hence there must be just one summand in the decomposition of $F$ given by (39).

To compute $H^{0}($ End $F) \cong H^{0}\left(F \otimes F^{*}\right)$ let us tensor (35) with $F^{*}$. We obtain the short exact sequence

$$
0 \longrightarrow p^{*} E_{1} \otimes F^{*} \longrightarrow F \otimes F^{*} \longrightarrow p^{*} E_{2} \otimes q^{*} \mathcal{O}(2) \otimes F^{*} \longrightarrow 0,
$$

and the corresponding sequence in cohomology

$$
0 \longrightarrow H^{0}\left(p^{*} E_{1} \otimes F^{*}\right) \longrightarrow H^{0}\left(F \otimes F^{*}\right) \longrightarrow H^{0}\left(p^{*} E_{2} \otimes q^{*} \mathcal{O}(2) \otimes F^{*}\right) \longrightarrow .
$$

We first compute $H^{0}\left(p^{*} E_{1} \otimes F^{*}\right)$. Dualizing (35), tensoring with $p^{*} E_{1}$, and using that $H^{0}\left(p^{*}\left(E_{1} \otimes E_{2}^{*}\right) \otimes q^{*} \mathcal{O}(-2)\right)=0$, we have the sequence in cohomology

$$
0 \longrightarrow H^{0}\left(p^{*} E_{1} \otimes F^{*}\right) \longrightarrow H^{0}\left(p^{*}\left(E_{1} \otimes E_{1}^{*}\right)\right) \stackrel{g}{\longrightarrow} H^{1}\left(p^{*}\left(E_{1} \otimes E_{2}^{*}\right) \otimes q^{*} \mathcal{O}(-2)\right) .
$$

By the Künneth formula

$H^{0}\left(p^{*}\left(E_{1} \otimes E_{1}^{*}\right)\right) \cong H^{0}\left(E_{1} \otimes E_{1}^{*}\right) \quad$ and $\quad H^{1}\left(p^{*}\left(E_{1} \otimes E_{2}^{*}\right) \otimes q^{*} \mathcal{O}(-2)\right) \cong H^{0}\left(E_{1} \otimes E_{2}^{*}\right)$.

Now, thanks to these isomorphisms, $g$ can be interpreted as the map $H^{0}\left(E_{1} \otimes E_{1}^{*}\right) \rightarrow$ $H^{0}\left(E_{1} \otimes E_{2}^{*}\right)$ defined by $\Phi$, i.e. $g(u)=u \Phi$. 
Now, from the $\tau$-stability of $\left(E_{1}, E_{2}, \Phi\right)$ one has from Corollary 3.10 that $\left(E_{1}, E_{2}, \Phi\right)$ is simple. Thus Ker $g \cong 0$ and from the exactness of (41) one obtains

$$
H^{0}\left(p^{*} E_{1} \otimes F^{*}\right)=0 .
$$

To compute $H^{0}\left(p^{*} E_{2} \otimes q^{*} \mathcal{O}(2) \otimes F^{*}\right)$, we dualize (35) and tensor it with $p^{*} E_{2} \otimes q^{*} \mathcal{O}(2)$, to get the sequence

$$
0 \longrightarrow H^{0}\left(p^{*}\left(E_{2} \otimes E_{2}^{*}\right)\right) \longrightarrow H^{0}\left(p^{*} E_{2} \otimes q^{*} \mathcal{O}(2) \otimes F^{*}\right) \longrightarrow H^{0}\left(p^{*}\left(E_{1}^{*} \otimes E_{2}\right) \otimes q^{*} \mathcal{O}(2)\right) .
$$

Lemma 4.4 Let $\left(E_{1}, E_{2}, \Phi\right)$ be $\tau$-stable and suppose that $\Phi$ is not an isomorphism, then $H^{0}\left(E_{1}^{*} \otimes E_{2}\right)=0$.

Proof. Suppose that there is a non-zero homomorphism $\Psi: E_{1} \rightarrow E_{2}$. Let $u=\Phi \Psi \in$ $H^{0}\left(\right.$ End $\left.E_{1}\right)$ and $v=\Psi \Phi \in H^{0}\left(\right.$ End $\left.E_{2}\right)$. Then $u \Phi=\Phi v$ and, since $\left(E_{1}, E_{2}, \Phi\right)$ is simple, we have that

$$
u=\lambda I_{E_{1}} \quad \text { and } \quad v=\lambda I_{E_{2}}, \quad \text { for } \quad \lambda \in C .
$$

If $\lambda \neq 0$, we easily see that $\Phi$ is an isomorphism, contradicting the assumption of the Lemma. Thus $\lambda=0$ and then

$$
\operatorname{Im} \Psi \subseteq \operatorname{Ker} \Phi \quad \text { and } \quad \operatorname{Im} \Phi \subseteq \operatorname{Ker} \Psi .
$$

We can therefore consider the subtriples of $\left(E_{1}, E_{2}, \Phi\right)$

$$
T_{1}=\left(K, E_{2}, \Phi\right) \quad \text { and } \quad T_{2}=(0, I, \Phi),
$$

where $K=\operatorname{Ker} \Psi$ and $I=\operatorname{Im} \Psi$. Let $r_{1}^{\prime}=\operatorname{rank} K, r_{1}^{\prime \prime}=\operatorname{rank} I, d_{1}^{\prime}=\operatorname{deg} K$ and $d_{1}^{\prime \prime}=\operatorname{deg} I$. Applying the $\tau$-stability condition to $T_{1}$ and $T_{2}$ we get the inequalities

$$
\begin{aligned}
& r_{2} d_{1}^{\prime \prime}-r_{1}^{\prime \prime}\left(d_{1}+d_{2}\right)+r_{1} r_{1}^{\prime \prime} \tau<0 \\
& d_{1}^{\prime}-d_{1}+\left(r_{1}-r_{1}^{\prime}\right) \tau<0 .
\end{aligned}
$$

From this and using that from

$$
0 \longrightarrow K \longrightarrow E_{1} \longrightarrow I \longrightarrow 0,
$$

$r_{1}=r_{1}^{\prime}+r_{1}^{\prime \prime}$ and $d_{1}=d_{1}^{\prime}+d_{1}^{\prime \prime}$, we obtain that

$$
\tau<\mu\left(E_{1} \oplus E_{2}\right),
$$

which is equivalent to $\sigma(\tau)<0$, contradicting the $\tau$-stability of $\left(E_{1}, E_{2}, \Phi\right)$.

From the Künneth formula and Lemma 4.4 we get that

$$
H^{0}\left(p^{*}\left(E_{1}^{*} \otimes E_{2}\right) \otimes q^{*} \mathcal{O}(2)\right) \cong H^{0}\left(E_{1}^{*} \otimes E_{2}\right) \otimes H^{0}(\mathcal{O}(2)) \cong 0,
$$


and from (43)

$$
H^{0}\left(p^{*} E_{2} \otimes q^{*} \mathcal{O}(2) \otimes F^{*}\right) \cong H^{0}\left(E_{2} \otimes E_{2}^{*}\right)
$$

From this and (42) the first three terms in (40) reduce to

$$
0 \longrightarrow H^{0}\left(F \otimes F^{*}\right) \stackrel{i}{\longrightarrow} H^{0}\left(E_{2} \otimes E_{2}^{*}\right)
$$

Since $F$ is $S U(2)$-invariantly stable then it is $S U(2)$-invariantly simple, i.e. the only $S U(2)$-invariant endomorphisms are multiples of the identity. Let $\Psi \in H^{0}\left(F \otimes F^{*}\right)$ be a non $S U(2)$-invariant endomorphism of $F$, i.e. $\Psi^{g} \neq \Psi$ for some $g \in S U(2)$. Since $i$ must be compatible with the action of $S U(2)$, we get

$$
i\left(\Psi^{g}\right)=(i(\Psi))^{g}
$$

On the other hand, since the action of $S U(2)$ on $H^{0}\left(E_{2} \otimes E_{2}^{*}\right)$ is trivial

$$
(i(\Psi))^{g}=i(\Psi)
$$

hence $i(\Psi)=i\left(\Psi^{g}\right)$ contradicting the injectivity of $i$. Thus $H^{0}\left(F \otimes F^{*}\right) \cong C^{*}$, which concludes the proof of our theorem for the case where $E_{1}$ and $E_{2}$ are not isomorphic.

Now suppose that $E_{1} \cong E_{2}$. We first prove

Lemma 4.5 Suppose $E_{1} \cong E_{2}$. Then for any $\tau>\mu\left(E_{1}\right)$, the triple $\left(E_{1}, E_{2}, \Phi\right)$ is $\tau$ stable if and only if $\Phi$ is an isomorphism and $E_{1}$ is stable.

Proof. Suppose that the triple is $\tau$-stable. Then (by Corollary 3.8) $\Phi$ is an isomorphism. Now consider the subtriples of the form $T^{\prime}=\left(\Phi\left(E_{2}^{\prime}\right), E_{2}^{\prime}, \Phi\right)$. These have $r_{1}^{\prime}=r_{2}^{\prime}$ and, since $\Phi$ is an isomorphism, $\mu\left(T^{\prime}\right)=\mu\left(E_{2}^{\prime}\right)$. Hence $\theta_{\tau}\left(T^{\prime}\right)=\mu\left(E_{2}^{\prime}\right)-\mu\left(E_{2}\right)$, and thus the $\tau$-stability of the triple implies the stability of $E_{2}$. Conversely, if $E_{2} \cong E_{1}$ and both are stable, then all non trivial $\Phi$ are isomorphisms. It now follows as a special case of Proposition 3.6 that the triple $\left(E_{1}, E_{2}, \Phi\right)$ is $\tau$-stable.

Suppose that $E_{1} \cong E_{2} \cong E$ and that the bundle $F$ associated to $(E, E, \Phi)$ is of the form

$$
F=p^{*} E \otimes q^{*} \mathcal{O}(1) \oplus p^{*} E \otimes q^{*} \mathcal{O}(1)
$$

If we assume now that $p^{*} E \otimes q^{*} \mathcal{O}(1)$ is stable, then $E$ is also stable and hence $H^{0}(E \otimes$ $\left.E^{*}\right) \cong C$. Thus there is only one non-trivial extension class (corresponding to $\Phi=\lambda I$ ). We must now examine this (unique) non-trivial extension

$$
0 \longrightarrow p^{*} E \longrightarrow F \longrightarrow p^{*} E \otimes q^{*} \mathcal{O}(\in) \longrightarrow \text { ' }
$$

This is of course nothing else but the pull-back to $X \times P^{1}$ of the non-trivial extension on $P^{1}$

$$
0 \longrightarrow \mathcal{O} \longrightarrow \mathcal{O}(1) \oplus \mathcal{O}(1) \longrightarrow \mathcal{O}(2) \longrightarrow 0
$$


tensored with $p^{*} E$. Thus the action of $S U(2)$ permutes the two summands in (44) and from Theorem 2.17 we conclude that $F$ is an $S U(2)$-invariantly stable bundle. The $\tau$-stability of $\left(E_{1}, E_{2}, \Phi\right)$ follows now from Proposition 4.2 .

Conversely, suppose that $\left(E_{1}, E_{2}, \Phi\right)$ is $\tau$-stable, then from Lemma 4.5 we obtain that $E_{1} \cong E_{2} \cong E$ is stable and $\Phi$ is hence a non-trivial multiple of the identity. From the above discussion we conclude that

$$
F=p^{*} E \otimes q^{*} \mathcal{O}(1) \oplus p^{*} E \otimes q^{*} \mathcal{O}(1)
$$

On the other hand, from Proposition 4.2, we argue as before that $F$ is certainly invariantly stable. Also (40), (41) and (42) show that we have an exact sequence

$$
0 \longrightarrow H^{0}\left(F \otimes F^{*}\right) \longrightarrow H^{0}\left(p^{*} E \otimes q^{*} \mathcal{O}(\in) \otimes \mathcal{F}^{*}\right)
$$

Using that $H^{0}\left(E \otimes E^{*}\right) \cong C$, the exact sequence (43) becomes,

$$
0 \longrightarrow C \longrightarrow H^{0}\left(p^{*} E \otimes q^{*} \mathcal{O}(\in) \otimes \mathcal{F}^{*}\right) \longrightarrow \mathcal{C}^{\ni}
$$

From these two exact sequences, we see that

$$
1 \leq h^{0}\left(F \otimes F^{*}\right) \leq h^{0}\left(p^{*} E \otimes q^{*} \mathcal{O}(\in) \otimes \mathcal{F}^{*}\right) \leq \triangle
$$

But since $F$ is invariantly stable it is given by the direct sum (39). In that case,

$$
H^{0}\left(F \otimes F^{*}\right) \cong G L(k, C),
$$

where $k$ is the number of stable summands in $F$. It follows that since $h^{0}\left(F \otimes F^{*}\right) \neq 1$, then $h^{0}\left(F \otimes F^{*}\right)=k^{2}-1$ for some integer $k$. The only possibility consistent with the constraint (45) is thus $h^{0}\left(F \otimes F^{*}\right)=3$, i.e. $k=2$. Hence the bundle $p^{*} E \otimes q^{*} \mathcal{O}(1)$ in the decomposition of $F$ is stable, which finishes the proof of the Theorem.

Notice that the conclusion of Proposition 4.2 extends straightforwardly to cover polystable objects. We thus get the following corollary, which will be useful in the next section

Theorem 4.6 Let $\left(E_{1}, E_{2}, \Phi\right)$ be a holomorphic triple and $F$ be the corresponding holomorphic bundle over $X \times P^{1}$. Let $\tau$ and $\sigma$ be related as above. Then $\left(E_{1}, E_{2}, \Phi\right)$ is $\tau$-polystable if and only if $F$ is $S U(2)$-invariantly polystable with respect to $\omega_{\sigma}$.

\section{$5 \quad$ Relation to vortex equations}

In this section we relate the $\tau$-stability of a triple to the existence of solutions to the coupled $\tau$-vortex equations. Using the idea of dimensional reduction this can be viewed as simply a special case of the Hitchin-Kobayashi correspondence between stable 
bundles and Hermitian-Einstein metrics. Indeed, specializing to the case of SU(2)equivariant bundles over $X \times P^{1}$ as in $\S 2$ (i.e. to the case of the bundles associated with triples over $X$ ), we have already assembled seen the results we need.

From GP3 (cf. also Theorem 2.18) we know that the SU(2)-equivariant bundle $F$ admits a Hermitian-Einstein metric with respect to $\omega_{\sigma}$ if and only if $F$ is polystable with respect to $\omega_{\sigma}$. By Theorem 4.6 we know that $F$ is $S U(2)$ invariantly polystable with respect to $\omega_{\sigma}$ if and only if the corresponding triple $\left(E_{1}, E_{2}, \Phi\right)$ is $\tau$-polystable with $\tau$ and $\sigma$ related by (37). Finally, by Proposition 2.14 there is a bijective correspondence between the $\mathrm{SU}(2)$-equivariant Hermitian-Einstein metrics on $F$ and the solutions to the coupled vortex equations (10) on $\left(E_{1}, E_{2}, \Phi\right)$.

Putting all this together, we see that we have filled in three sides of the following "commutative diagram" for a holomorphic triple over $X$ and the corresponding SU(2)equivariant bundle over $X \times P^{1}$ (with Kähler form $\omega_{\sigma}$ )

\begin{tabular}{|c|c|c|c|}
\hline & $\begin{array}{l}\text { SPECIAL } \\
\text { METRICS }\end{array}$ & $\begin{array}{c}\text { Hitchin-Kobayashi } \\
\text { correspondence }\end{array}$ & $\begin{array}{c}\text { HOLOMORPHIC } \\
\text { INTERPRETATION }\end{array}$ \\
\hline On $X \times P^{1}$ & $\begin{array}{c}\text { SU(2)-invariant Solutions } \\
\text { to Hermitian-Einstein } \\
\text { Equations }\end{array}$ & Th. 2.18 & $\begin{array}{l}\text { SU(2)-invariantly } \\
\text { Polystable } \\
\text { Extensions }\end{array}$ \\
\hline dim. red. & Prop. 2.14 & & Th. 4.6 \\
\hline On $X$ & $\begin{array}{l}\text { Solutions to } \\
\text { Coupled } \tau \text {-Vortex } \\
\text { Equations }\end{array}$ & & $\begin{array}{c}\tau \text {-stable } \\
\text { holomorphic } \\
\text { Triples }\end{array}$ \\
\hline
\end{tabular}

By tracing around the three sides of this diagram which already have arrows filled in, we can fill in the arrows on the fourth side and prove

Theorem 5.1 Let $T=\left(E_{1}, E_{2}, \Phi\right)$ be a holomorphic triple. Then the following are equivalent.

(1) The bundles support Hermitian metrics $h_{1}, h_{2}$ such that the coupled $\tau$-vortex equations are satisfied, i.e. such that

$$
\begin{aligned}
& \sqrt{-1} \Lambda F_{h_{1}}+\Phi \Phi^{*}=2 \pi \tau I_{E_{1}} \\
& \sqrt{-1} \Lambda F_{h_{2}}-\Phi^{*} \Phi=2 \pi \tau^{\prime} I_{E_{2}}
\end{aligned}
$$

with

$$
r_{1} \tau+r_{2} \tau^{\prime}=\operatorname{deg} E_{1}+\operatorname{deg} E_{2},
$$

(2) The triple is $\tau$-polystable.

Notice that the statement and conclusion of this theorem make no mention of $X \times P^{1}$ or the $S U(2)$-equivariant bundle $F$. One might thus expect a more direct 
proof that does not use dimensional reduction. We will not attempt to prove both directions of the biconditional in the theorem in this way, but the one direction is quite easily seen. That is, one can show how the $\tau$-stability condition can be derived directly as a consequence of the coupled vortex equations. We do this as follows.

Let $T^{\prime}=\left(E_{1}^{\prime}, E_{2}^{\prime}, \Phi\right)$ be a holomorphic saturated sub-triple of $T$, and let

$$
E_{1}=E_{1}^{\prime} \oplus E_{1}^{\prime \prime}
$$

and

$$
E_{2}=E_{2}^{\prime} \oplus E_{2}^{\prime \prime}
$$

be smooth orthogonal splittings of $E_{1}$ and $E_{2}$. With respect to these splittings, we get a block diagonal decomposition of $\sqrt{-1} \Lambda F_{h_{1}}, \sqrt{-1} \Lambda F_{h_{2}}$ as

$$
\sqrt{-1} \Lambda F_{h_{i}}=\left(\begin{array}{cc}
\sqrt{-1} \Lambda F_{i}^{\prime}+\Pi_{i} & * \\
* & \sqrt{-1} \Lambda F_{i}^{\prime \prime}-\Pi_{i}
\end{array}\right)
$$

where $\Pi_{i}$ is a positive definite endomorphism coming from the second fundamental form for the inclusion of $E_{i}^{\prime}$ in $E_{i}$. We also get a decomposition of $\Phi$ as

$$
\Phi=\left(\begin{array}{cc}
\Phi^{\prime} & \Theta \\
0 & \Phi^{\prime \prime}
\end{array}\right) .
$$

The coupled vortex equations thus split into equations on the summands of $E_{1}$ and $E_{2}$ to yield

$$
\begin{aligned}
\sqrt{-1} \Lambda F_{1}^{\prime}+\Pi_{1}+\Phi^{\prime}\left(\Phi^{\prime}\right)^{*}+\Theta \Theta^{*} & =2 \pi \tau I_{1}^{\prime}, \\
\sqrt{-1} \Lambda F_{1}^{\prime \prime}-\Pi_{1}+\Phi^{\prime \prime}\left(\Phi^{\prime \prime}\right)^{*} & =2 \pi \tau I_{1}^{\prime \prime}, \\
\sqrt{-1} \Lambda F_{2}^{\prime}+\Pi_{2}-\left(\Phi^{\prime}\right)^{*} \Phi^{\prime} & =2 \pi \tau^{\prime} I_{2}^{\prime}, \\
\sqrt{-1} \Lambda F_{2}^{\prime \prime}-\Pi_{2}-\left(\Phi^{\prime \prime}\right)^{*} \Phi^{\prime \prime}-\Theta^{*} \Theta & =2 \pi \tau^{\prime} I_{2}^{\prime \prime} .
\end{aligned}
$$

We now integrate the trace of these equations over the base manifold $X$. We use the notation

$$
\begin{gathered}
d_{i}^{\prime}=\operatorname{deg} E_{i}^{\prime}=\frac{\sqrt{-1}}{2 \pi} \int_{X} \operatorname{Tr}\left(\Lambda F_{i}^{\prime}\right), \\
d_{i}^{\prime \prime}=\operatorname{deg} E_{i}^{\prime \prime}=\frac{\sqrt{-1}}{2 \pi} \int_{X} \operatorname{Tr}\left(\Lambda F_{i}^{\prime \prime}\right), \\
\left\|\Pi_{i}\right\|^{2}=\frac{1}{2 \pi} \int_{X} \operatorname{Tr}\left(\Pi_{i}\right), \\
\|\Theta\|^{2}=\frac{1}{2 \pi} \int_{X} \operatorname{Tr}\left(\Theta \Theta^{*}\right),
\end{gathered}
$$

From equations (46) and (48) we get

$$
d_{1}^{\prime \prime}+d_{2}^{\prime \prime}-\left(\left\|\Pi_{1}\right\|^{2}+\left\|\Pi_{2}\right\|^{2}\right)-\|\Theta\|^{2}=\tau\left(r_{1}-r_{1}^{\prime}\right)+\tau^{\prime}\left(r_{2}-r_{2}^{\prime}\right),
$$


and from (47) and (51) we get

$$
d_{1}^{\prime}+d_{2}^{\prime}+\left(\left\|\Pi_{1}\right\|^{2}+\left\|\Pi_{2}\right\|^{2}\right)+\|\Theta\|^{2}=\tau r_{1}^{\prime}+\tau^{\prime} r_{2}^{\prime}
$$

Using the fact that $d_{i}=d_{i}^{\prime}+d_{i}^{\prime \prime}$, these equations can be combined to give

$$
\operatorname{deg}\left(E_{1}^{\prime} \oplus E_{2}^{\prime}\right)+2\left(\left\|\Pi_{1}\right\|^{2}+\left\|\Pi_{2}\right\|^{2}+\|\Theta\|^{2}\right)=r_{1}^{\prime} \tau+r_{2}^{\prime} \tau^{\prime}
$$

It now follows by the positivity of the terms $\left\|\Pi_{i}\right\|^{2}$ and $\|\Theta\|^{2}$, that

$$
\mu\left(T^{\prime}\right) \leq \frac{r_{1}^{\prime} \tau+r_{2}^{\prime} \tau^{\prime}}{r_{1}^{\prime}+r_{2}^{\prime}}
$$

But from equation (48) we have that

$$
\frac{r_{1} \tau+r_{2} \tau^{\prime}}{r_{1}+r_{2}}=\mu(T)
$$

and by using this to solve for $\tau^{\prime}$, one sees that (58) is equivalent to the condition $\theta_{\tau}\left(T^{\prime}\right) \leq 0$. Furthermore, it follows from (57) that in order to have $\theta_{\tau}\left(T^{\prime}\right)=0$, one needs

$$
\left\|\Pi_{1}\right\|^{2}=\left\|\Pi_{2}\right\|^{2}=\|\Theta\|^{2}=0 .
$$

This means that the bundles split holomorphically as $E_{i}=E_{i}^{\prime} \oplus E_{i}^{\prime \prime}$ and $\Phi=\Phi^{\prime} \oplus \Phi^{\prime \prime}$, i.e. the triple $T=\left(E_{1}, E_{2}, \Phi\right)$ splits as $T=\left(E_{1}^{\prime}, E_{2}^{\prime}, \Phi^{\prime}\right) \oplus\left(E_{1}^{\prime \prime}, E_{2}^{\prime \prime}, \Phi^{\prime \prime}\right)$. Each summand separately supports a solution to the coupled $\tau$-vortex equations. It is possible that in this splitting $\Phi^{\prime}$ or $\Phi^{\prime \prime}$ is trivial. The bundles in the degenerate subtriple then each supports solutions to the Hermitian-Einstein equations. We have thus proven the following proposition:

Proposition 5.2 Let $T=\left(E_{1}, E_{2}, \Phi\right)$ be a holomorphic triple in which the bundles support Hermitian metrics $h_{1}, h_{2}$ such that the coupled $\tau$-vortex equations are satisfied. Then

(1) $T$ splits as a direct sum of triples $\left(E_{1 i}, E_{2 i}, \Phi_{i}\right)$, i.e. $E_{1}=\bigoplus E_{1 i}, E_{2}=\bigoplus E_{2 i}$, and $\Phi=\bigoplus \Phi_{i}$

(2) each summand $\left(E_{1 i}, E_{2 i}, \Phi_{i}\right)$ is either a $\tau$-stable triple, or $\Phi_{i}=0$ and both bundles are stable. In the latter case, the slope of $E_{1 i}$ is $\tau$ and the slope of $E_{2 i}$ is $\tau^{\prime}$.

That is $T$ is $\tau$-polystable.

\section{Moduli spaces}




\subsection{Moduli spaces of stable triples}

Recall that two triples $T=\left(E_{1}, E_{2}, \Phi\right)$ and $T^{\prime}=\left(E_{1}^{\prime}, E_{2}^{\prime}, \Phi^{\prime}\right)$ are isomorphic if there exist isomorphisms $u: E_{1} \longrightarrow E_{1}^{\prime}$ and $v: E_{2} \longrightarrow E_{2}^{\prime}$ making the following diagram commutative

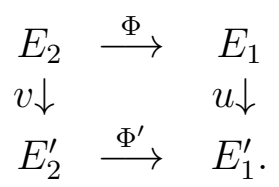

After fixing the topological invariants of our bundles, that is the ranks $r_{1}$ and $r_{2}$ and the first Chern classes $d_{1}$ and $d_{2}$, let $\mathfrak{M}$ be the set of equivalence classes of holomorphic triples and $\mathfrak{M}_{\tau} \subset \mathfrak{M}$ be the subset of equivalence classes of $\tau$-stable triples. Our goal in this section is to show that $\mathfrak{M}_{\tau}$ has the structure of an algebraic variety, more precisely:

Theorem 6.1 Let $X$ be a compact Riemann surface of genus $g$ and let us fix ranks $r_{1}$ and $r_{2}$ and degrees $d_{1}$ and $d_{2}$. The moduli space of $\tau$-stable triples $\mathfrak{M}_{\tau}$ is a complex analytic space with a natural Kähler structure outside of the singularities. Its dimension at a smooth point is

$$
1+r_{2} d_{1}-r_{1} d_{2}+\left(r_{1}^{2}+r_{2}^{2}-r_{1} r_{2}\right)(g-1)
$$

The moduli space, $\mathfrak{M}_{\tau}$ is non-empty if and only if $\tau$ is inside the interval

$$
\left(\mu\left(E_{1}\right), \mu_{M A X}\right)
$$

where

$$
\mu_{M A X}=\mu\left(E_{1}\right)+\frac{r_{2}}{\left|r_{1}-r_{2}\right|}\left(\mu\left(E_{1}\right)-\mu\left(E_{2}\right)\right)
$$

if $r_{1} \neq r_{2}$, and $\mu_{M A X}=\infty$ if $r_{1}=r_{2}$.

Moreover $\mathfrak{M}_{\tau}$ is in general a quasi-projective variety. It is in fact projective if $r_{1}+r_{2}$ and $d_{1}+d_{2}$ are coprime and $\tau$ is generic.

Proof. There are several approaches one can take to prove this theorem. One can use standard Kuranishi deformation methods as done in [B-D1, B-D2 for the construction of the moduli spaces of stable pairs. Alternatively one can use geometric invariant theory methods to give an algebraic geometric construction of our moduli spaces, generalizing the construction of the moduli space of stable pairs given in $[\mathbb{B}, \mathbb{1}]$. We will leave these two direct methods for a future occasion and instead will exploit the relation between $\tau$-stable triples and equivariant bundles over $X \times P^{1}$. This method, which is used in GP3 to construct the moduli spaces of triples when $E_{2}$ is a line bundle, leads also to an alternative construction of the moduli spaces of stable pairs. Apart from smoothness considerations, which we shall discuss later, the arguments of the proof are the same that those in GP3. 
Let $\sigma$ be related to $\tau$ by (37) and let $\omega_{\sigma}$ be the Kähler form on $X \times P^{1}$ defined by (36). Let $\mathcal{M}_{\sigma}$ be the moduli space of stable bundles with respect to $\omega_{\sigma}$ whose underlying smooth bundle is defined by (1). Let us exclude for the moment the case $r_{1}=r_{2}$ and $d_{1}=d_{2}$. Let $F \longrightarrow X \times P^{1}$ be the bundle associated to $\left(E_{1}, E_{2}, \Phi\right)$ as in Proposition 2.4. Theorem 4.1 says that the correspondence $\left(E_{1}, E_{2}, \Phi\right) \longmapsto F$ defines a map

$$
\mathfrak{M}_{\tau} \longrightarrow \mathcal{M}_{\sigma}
$$

The action of $S U(2)$ on $X \times P^{1}$ defined in Section 2 induces an action on $\mathcal{M}_{\sigma}$ and, since the bundle $F$ associated to $\left(E_{1}, E_{2}, \Phi\right)$ is $S U(2)$-equivariant the image of the above map is contained in $\mathcal{M}_{\sigma}^{S U(2)}$ - the set of fixed points of $\mathcal{M}_{\sigma}$ under the $S U(2)$ action. As proved in GP3, Proposition 5.3] the set $\mathcal{M}_{\sigma}^{S U(2)}$ can be described as a disjoint union of a finite number of sets

$$
\mathcal{M}_{\sigma}^{S U(2)}=\bigcup_{i \in I} \mathcal{M}_{\sigma}^{i}
$$

The index $I$ ranges over the set of equivalence classes of different smooth $S U(2)$ equivariant structures on the smooth bundle $F$ defined by (11). Of course the way of writing $F$ in (11) already exhibits a particular $S U(2)$-equivariant structure, but in principle the bundle $F$ might admit different ones. The set $\mathcal{M}_{\sigma}^{i}$ corresponds to the set of equivalence classes in $\mathcal{M}_{\sigma}$ admitting a representative which is $S U(2)$-equivariant for the smooth equivariant structure defined by $i \in I$. An equivariant smooth structure defines an action on the space of smooth automorphisms of the bundle $F$ and, as shown in [GP3, Theorem 5.6] the sets $\mathcal{M}_{\sigma}^{i}$ can be described as the set of equivalence classes of $S U(2)$-equivariant holomorphic structures on the underlying smooth $S U(2)$-equivariant bundle defined by $i$, modulo $S U(2)$-equivariant isomorphisms.

Let $i_{0}$ be the $C^{\infty} S U(2)$-equivariant structure on $F$ defined by (1). As shown in Proposition 2.4 there is a one-to-one correspondence

$$
\text { \{holomorphic triples }\} \stackrel{1-1}{\longleftrightarrow}\left\{i_{0} \text {-equivariant holomorphic vector bundles }\right\} \text {. }
$$

On the other hand by Lemma 2.10 the equivariant homomorphisms between two equivariant holomorphic bundles $F$ and $F^{\prime}$ corresponding to triples $T$ and $T^{\prime}$, respectively, are in one-to-one correspondence with the morphisms between $T$ and $T^{\prime}$. In fact the correspondence (62) descends to the quotient and thus from Theorem 4.1 we can identify $\mathfrak{M}_{\tau}$ with $\mathcal{M}_{\sigma}^{i_{0}}$. The properties of $\mathfrak{M}_{\tau}$ follow now from standard facts about the more familiar moduli spaces of stable bundles $\mathcal{M}_{\sigma}[\mathrm{D}-\mathrm{K}, \mathrm{G}, \mathrm{M}, \mathrm{Ko}$, and more particularly of the fixed-point sets $\mathcal{M}_{\sigma}^{i}$ (see [GP3, Theorem 5.6] for details). Namely,

Theorem 6.2 $\mathcal{M}_{\sigma}^{i}$ is a complex analytic variety. A point $[F] \in \mathcal{M}_{\sigma}^{i}$ is non-singular if it is non-singular as a point of $\mathcal{M}_{\sigma}$. The tangent space at such a point can be identified with the $S U(2)$-invariant part of $H^{1}\left(X \times P^{1}\right.$, End $\left.F\right) . \mathcal{M}_{\sigma}^{i}$ has a natural Kähler structure induced from that of $\mathcal{M}_{\sigma}$. Moreover if $\sigma$ is a rational number then $\mathcal{M}_{\sigma}^{i}$ is a quasi-projective variety. 
From this theorem and the identification of $\mathfrak{M}_{\tau}$ with $\mathcal{M}_{\sigma}^{i_{0}}$ we deduce that $\mathfrak{M}_{\tau}$ is a complex analytic variety with a Kähler metric outside the singularities. To compute the dimension of the tangent space at a smooth point $[T]$ it suffices to compute the dimension of the $S U(2)$-invariant part of $H^{1}\left(X \times P^{1}\right.$, End $\left.F\right)$. This can be done in a similar way to that of [GP3, Theorem 5.13] to obtain that

$$
\operatorname{dim} \mathfrak{M}_{\tau}=1+\chi\left(E_{1} \otimes E_{2}^{*}\right)-\chi\left(\text { End } E_{1}\right)-\chi\left(\text { End } E_{2}\right),
$$

which by Riemann-Roch yields (59).

We consider now the case $r_{1}=r_{2}=r$ and $d_{1}=d_{2}=d$. In this case by Lemma 4.5 we can identify the moduli space $\mathfrak{M}_{\tau}$ with the moduli space of stable bundles of rank $r$ and degree $d$ on $X$. The theorem follows now from well-known results about this moduli space $\mathrm{A}-\mathrm{B}, \mathrm{N}-\mathrm{S}$.

The fact that $\mathfrak{M}_{\tau}$ is empty outside the interval ([0) if $r_{1} \neq r_{2}$ and outside $\left(\mu\left(E_{1}\right), \infty\right)$ if $r_{1}=r_{2}$ follows from Proposition 3.14. As explained in Proposition 3.18 the nongeneric values divide this intervals in subintervals in such a way that the stability properties of a given triple do not change for two values of $\tau$ in the same subinterval. Therefore we can always choose $\tau$ (and hence $\sigma$ ) to be rational, which by Theorem 6.2 gives that $\mathfrak{M}_{\tau}$ is quasi-projective.

To show the compactness of $\mathfrak{M}_{\tau}$ when $r_{1}+r_{2}$ and $d_{1}+d_{2}$ are coprime and $\tau$ is generic (we are also assuming that $r_{1} \neq r_{2}$ or $d_{1} \neq d_{2}$ ) we consider a sequence of points in $\mathcal{M}_{\sigma}^{i_{0}}$. This sequence must converge in $\overline{\mathcal{M}_{\sigma}}$ - the Uhlenbeck compactification of $\mathcal{M}_{\sigma}$. Using $S U(2)$-invariance one can see that the limit has to correspond to a polystable element, but by Proposition 3.18 this has to be actually stable, that is the limit must be in $\mathcal{M}_{\sigma}$ and hence in $\mathcal{M}_{\sigma}^{i_{0}}$ since this is closed. The compactness when $r_{1}=r_{2}$ and $d_{1}=d_{2}$ follows from the compactness of the moduli space of stable bundles of rank $r$ and degree $d$ when $r$ and $d$ are coprime. The compactness of $\mathfrak{M}_{\tau}$ can also be obtained (as it is done for pairs in B-D1]) from the fact that it can be identified with the moduli space of solutions to the coupled vortex equations and these are moment map equations as we shall explain later.

It was shown in [GP3, Theorem 5.13] that when $E_{2}$ is a line bundle our moduli spaces are smooth for every value of $\tau$. This does not seem to be the case when $E_{2}$ is of arbitrary rank. However we can show the following

Proposition 6.3 Let $T=\left(E_{1}, E_{2}, \Phi\right)$ be a holomorphic triple such that $\Phi$ is either injective or surjective, then $[T]$ is a smooth point of $\mathfrak{M}_{\tau}$.

Proof. Let

$$
0 \longrightarrow p^{*} E_{1} \longrightarrow F \longrightarrow p^{*} E_{2} \otimes q^{*} \mathcal{O}(2) \longrightarrow 0,
$$

be the extension over $X \times P^{1}$ corresponding to $T$. To prove the smoothness of $\mathfrak{M}_{\tau}$ at the point $\left[\left(E_{1}, E_{2}, \Phi\right)\right]$ it suffices to show that $H^{2}\left(X \times P^{1}\right.$, End $\left.F\right)=0$. Tensoring (63) with $F^{*}$ the last terms in the corresponding long exact sequence are

$$
H^{2}\left(p^{*} E_{1} \otimes F^{*}\right) \longrightarrow H^{2}\left(F \otimes F^{*}\right) \longrightarrow H^{2}\left(p^{*} E_{2} \otimes q^{*} \mathcal{O}(2) \otimes F^{*}\right) \longrightarrow 0 .
$$


By Serre duality

$$
\begin{gathered}
H^{2}\left(p^{*} E_{1} \otimes F^{*}\right) \cong H^{0}\left(p^{*}\left(E_{1}^{*} \otimes K\right) \otimes F\right)^{*} \\
H^{2}\left(p^{*} E_{2} \otimes q^{*} \mathcal{O}(2) \otimes F^{*}\right) \cong H^{0}\left(p^{*}\left(E_{2}^{*} \otimes K\right) \otimes q^{*} \mathcal{O}(-4) \otimes F\right)^{*},
\end{gathered}
$$

where $K$ is the canonical line bundle of $X$.

It is easy to see that $H^{0}\left(p^{*}\left(E_{2}^{*} \otimes K\right) \otimes q^{*} \mathcal{O}(-4) \otimes F\right) \cong 0$. To analyse $H^{0}\left(p^{*}\left(E_{1}^{*} \otimes\right.\right.$ $K) \otimes F)$ we tensor $(63)$ with $p^{*}\left(E_{1}^{*} \otimes K\right) \otimes q^{*} \mathcal{O}(-2)$, and since $H^{0}\left(p^{*}\left(E_{1} \otimes E_{1} \otimes K\right) \otimes\right.$ $\left.q^{*} \mathcal{O}(-2)\right) \cong 0$, we obtain

$0 \longrightarrow H^{0}\left(p^{*}\left(E_{1}^{*} \otimes K\right) q^{*} \mathcal{O}(-2) \otimes F\right) \longrightarrow H^{0}\left(p^{*}\left(E_{1}^{*} \otimes E_{2} \otimes K\right)\right) \stackrel{f}{\longrightarrow} H^{1}\left(p^{*}\left(E_{1} \otimes E_{1}^{*} \otimes K \otimes \mathcal{O}(-2)\right)\right.$.

The map $f$ in the above sequence is essentially the map

$$
\begin{array}{ccc}
H^{0}\left(E_{1}^{*} \otimes E_{2} \otimes K\right) & \stackrel{f}{\longrightarrow} & H^{0}\left(E_{1} \otimes E_{1}^{*} \otimes K\right) \\
\Psi & \longmapsto & \Phi \circ \Psi .
\end{array}
$$

Assume now that $\Phi$ is injective, if we prove that $f$ is also injective, by the exactness of (65) we would be done. Suppose that Ker $f \neq 0$. This means that there exists a non-zero map $\Psi: E_{1} \longrightarrow E_{2} \otimes K$, and since $\Psi \circ \Phi=0, \operatorname{Im} \Psi$ is a non-trivial subsheaf contained in $\operatorname{Ker} \Phi$ contradicting the injectivity.

To prove smoothness when $\Phi$ is surjective, we consider the dual triple $T^{*}=\left(E_{2}^{*}, E_{1}^{*}, \Phi^{*}\right)$. $\Phi^{*}$ is now injective and the result follows from the fact that $T=\left(E_{1}, E_{2}, \Phi\right)$ is a smooth point if and only if $T^{*}=\left(E_{2}^{*}, E_{1}^{*}, \Phi^{*}\right)$ is a smooth point.

\subsection{Abel-Jacobi maps}

As shown in Proposition 3.19 there is a range for the parameter $\tau$ such that the $\tau$ stability of a triple $\left(E_{1}, E_{2}, \Phi\right)$ implies the semistability of $E_{1}$ and $E_{2}$. Let $\mathfrak{M}_{0}$ be the moduli space of $\tau$-stable triples for $\tau$ in such a range. Let $N(r, d)$ be the Seshadri compactification of the moduli space of stable bundles of rank $r$ and degree $d$ over $X$, that is, the space of $S$-equivalence classes of semistable bundles.

There are natural "Abel-Jacobi" maps $\pi_{1}$ and $\pi_{2}$

$$
\begin{aligned}
& \mathfrak{M}_{0} \quad \stackrel{\pi_{2}}{\longrightarrow} N\left(r_{2}, d_{2}\right) \\
& \quad \pi_{1} \downarrow \\
& N\left(r_{1}, d_{1}\right)
\end{aligned}
$$

defined as

$$
\pi_{1}\left(\left[\left(E_{1}, E_{2}, \Phi\right)\right]\right)=\left[E_{1}\right] \text { and } \pi_{2}\left(\left[\left(E_{1}, E_{2}, \Phi\right)\right]\right)=\left[E_{2}\right] .
$$

We know also from Proposition 3.19 that if both $E_{1}$ and $E_{2}$ are stable then the intersection of the fibres $\pi_{1}^{-1}\left(\left[E_{1}\right]\right)$ and $\pi_{2}^{-1}\left(\left[E_{2}\right]\right)$ can be identified with $P\left(H^{0}\left(E_{1} \otimes E_{2}^{*}\right)\right)$. In general, though, this intersection for non-stable points is hard to describe. 
If $\mu\left(E_{1} \otimes E_{2}^{*}\right)>2 g-2$, that is if

$$
r_{2} d_{1}-r_{1} d_{2}>r_{1} r_{2}(2 g-2)
$$

where $g$ is the genus of $X$, then $H^{1}\left(E_{1} \otimes E_{2}^{*}\right)=0$ for $E_{1}$ and $E_{2}$ stable and the projection from $\mathfrak{M}_{0}$ to $N\left(r_{1}, d_{1}\right) \times N\left(r_{2}, d_{2}\right)$ is a fibration on the the stable part.

Recall that if $\left(r_{1}, d_{1}\right)=1$ and $\left(r_{2}, d_{2}\right)=1$ then stability and semistability coincide and there exist universal bundles

$$
E_{1} \longrightarrow X \times N\left(r_{1}, d_{1}\right) \text { and } E_{2} \longrightarrow X \times N\left(r_{2}, d_{2}\right)
$$

Let us denote by $p_{1}, p_{2}$ and $\pi$ the projections from $X \times N\left(r_{1}, d_{1}\right) \times N\left(r_{2}, d_{2}\right)$ to $X \times$ $N\left(r_{1}, d_{1}\right), X \times N\left(r_{2}, d_{2}\right)$, and $N\left(r_{1}, d_{1}\right) \times N\left(r_{2}, d_{2}\right)$ respectively. It is clear that $\mathfrak{M}_{0}$ can be identified with

$$
P\left(\pi_{*}\left(p_{1}^{*} E_{1} \otimes p_{2}^{*} E_{2}^{*}\right)\right) .
$$

But in the non-coprime situation we have no universal bundles $E_{1}$ and $E_{2}$ available and the analogue of (66) has to be constructed as a moduli space in its own right.

As explained in Theorem 6.1 the moduli space of $\tau$-stable triples is non-empty if and only $\tau$ is in the interval $I=\left(\mu\left(E_{1}\right), \mu_{M A X}\right)$. We saw in $\$ 3.4$ that the stability properties of a given triple can change only at certain rational values of $\tau$ (the critical values) which divide $I$ in a finite number of subintervals. The moduli spaces for values of $\tau$ in the same open subinterval are then isomorphic, and they might change only when crossing one of the critical values. We expect that, as in the case of stable pairs [B-D-W], [T], the moduli spaces for consecutive intervals must be related by some sort of flip-type birational transformation. This, as well as the construction of a "master" space for triples (cf. [B-D-W]) containing the moduli space of triples for all possible values of $\tau$, will be dealt with in a future paper.

\subsection{Vortices}

Thanks to our existence theorem the moduli space of stable triples can be interpreted as the moduli space of solutions to the coupled vortex equations. To understand the meaning of this statement one needs to regard the vortex equations as equations for unitary connections instead of equations for metrics. This point of view corresponds to the fact that fixing a holomorphic structure and varying the metric on a vector bundle is equivalent to fixing the metric and varying the holomorphic structure - or the corresponding connection. Recall that the space of unitary connections on a smooth

Hermitian vector bundle can be identified with the space of $\bar{\partial}$-operators which in turn corresponds with the space of holomorphic structures on our bundle.

Let $E_{1}$ and $E_{2}$ be smooth vector bundles over $X$ and $h_{1}$ and $h_{2}$ be Hermitian metrics on $E_{1}$ and $E_{2}$ respectively. Let $\mathcal{A}_{1}$ (resp. $\mathcal{A}_{2}$ ) be the space of unitary connections on $\left(E_{1}, h_{1}\right)$ (resp. $\left.\left(E_{2}, h_{2}\right)\right)$. Let $\left(A_{1}, A_{2}, \Phi\right) \in \mathcal{A}_{1} \times \mathcal{A}_{2} \times \Omega^{0}\left(\operatorname{Hom}\left(E_{2}, E_{1}\right)\right)$. The vortex 
equations can be regarded as the equations for $\left(A_{1}, A_{2}, \Phi\right)$

$$
\left.\begin{array}{l}
\bar{\partial}_{A_{1 *} A_{2}} \Phi=0 \\
\sqrt{-1} \Lambda F_{A_{1}}+\Phi \Phi^{*}=2 \pi \tau I_{E_{1}} \\
\sqrt{-1} \Lambda F_{A_{2}}-\Phi^{*} \Phi=2 \pi \tau^{\prime} I_{E_{2}}
\end{array}\right\} .
$$

The connections $A_{1}$ and $A_{2}$ induce holomorphic structures on $E_{1}$ and $E_{2}$ and the first equation in (67) simply says that $\Phi$ must be holomorphic.

Let $\mathcal{G}_{1}$ and $\mathcal{G}_{2}$ be the gauge groups of unitary transformations of $\left(E_{1}, h_{1}\right)$ and $\left(E_{2}, h_{2}\right)$ respectively. $\mathcal{G}_{1} \times \mathcal{G}_{2}$ acts on $\mathcal{A}_{1} \times \mathcal{A}_{2} \times \Omega^{0}\left(\operatorname{Hom}\left(E_{2}, E_{1}\right)\right)$ by the rule

$$
\left(g_{1}, g_{2}\right) \cdot\left(A_{1}, A_{2}, \Phi\right)=\left(g_{1} A_{1} g_{1}^{-1}, g_{2} A_{2} g_{2}^{-1}, g_{1} \Phi g_{2}^{-1}\right)
$$

The action of $\mathcal{G}_{1} \times \mathcal{G}_{2}$ preserves the equations and the moduli space of coupled $\tau$-vortices is defined as the space of all solutions to (67) modulo this action.

The moduli space of vortices can be obtained as a symplectic reduction (see GP3, Section 2.2]) in a similar way to the moduli space of Hermitian-Einstein connections: $\mathcal{A}_{1} \times \mathcal{A}_{2} \times \Omega^{0}\left(\operatorname{Hom}\left(E_{2}, E_{1}\right)\right)$ admits a Kähler structure which is preserved by the action of $\mathcal{G}_{1} \times \mathcal{G}_{2}$. Associated to this action there is a moment map given precisely by

$$
\left(A_{1}, A_{2}, \Phi\right) \longmapsto\left(\Lambda F_{A_{1}}-\sqrt{-1} \Phi \Phi^{*}+2 \sqrt{-1} \pi \tau, \Lambda F_{A_{2}}+\sqrt{-1} \Phi^{*} \Phi+2 \sqrt{-1} \pi \tau^{\prime}\right) .
$$

Let $\mu$ be this moment map restricted to the subvariety

$$
\mathcal{N}=\left\{\left(A_{1}, A_{2}, \Phi\right) \in \mathcal{A}_{1} \times \mathcal{A}_{2} \times \Omega^{0}\left(\operatorname{Hom}\left(E_{2}, E_{1}\right)\right) \mid \bar{\partial}_{A_{1} * A_{2}} \Phi=0\right\}
$$

The moduli space of $\tau$-vortices is then nothing else but the symplectic quotient

$$
\mu^{-1}(0,0) / \mathcal{G}_{1} \times \mathcal{G}_{2}
$$

and Theorem 5.1 can be reformulated by saying that there is a one-to-one correspondence

$$
\mu^{-1}(0,0) / \mathcal{G}_{1} \times \mathcal{G}_{2} \stackrel{1-1}{\longrightarrow} \mathfrak{M}_{\tau}
$$

\section{Some generalizations}

1. Although for simplicity we have worked on a Riemann surface, most of our results extend in a straightforward manner to a compact complex manifold of arbitrary dimension. Of course, as in ordinary stability, one needs to choose a Kähler metric in order to define the degree of a coherent sheaf and hence the slopes involved in the definition of $\tau$-stability for a triple.

2. One of our main goals in this paper has been to show that our stability condition for a triple corresponds to the existence of solutions to the coupled vortex equations. This is the main reason for defining our stability criterium only for vector bundles. 
One can more generally define $\tau$-stability for a triple consisting of two (torsion free) coherent sheaves and a morphism between them. The main results of Sections 3 and 4 go through in this more general situation.

3. A. King [K] has been able to characterize all $S U(2)$-equivariant holomorphic vector bundles on $X \times P^{1}$. Generalizing the results in Section 2, he has shown that these bundles are in one-to-one correspondence with $(2 n-1)$-tuples consisting of $n$ holomorphic vector bundles $E_{1}, \ldots, E_{n}$ over $X$ and a chain of morphisms

$$
E_{n} \stackrel{\Phi_{n-1}}{\longrightarrow} E_{n-1} \longrightarrow \ldots \longrightarrow E_{2} \stackrel{\Phi_{1}}{\longrightarrow} E_{1}
$$

He has defined a stability condition for such a $(2 n-1)$-tuple which involves $(n-1)$ parameters and that specializes to our stability condition for a triple when $n=2$. In fact he considers this notion for more general diagrams than the one above. Presumably this stability condition governs, as for triples, the existence of Hermitian metrics on the bundles $E_{i}$ satisfying some generalized vortex equations naturally associated to the $(2 n-1)$-tuple.

4. Our results have also been extended in a different direction $\mathbb{B}-\mathrm{GP}$ to parabolic triples, that is to triples in which the bundles are endowed with parabolic structures. The Higgs field can be either a parabolic morphism or a meromorphic morphism with simple poles at the parabolic points and whose residues respect the parabolic structure in some precise sense. In both cases one can prove a Hitchin-Kobayashi correspondence, although the metrics involved now have singularities at the parabolic points.

Acknowledgements. The authors would like to thank Alastair King and Jun Li for helpful conversations and the following institutions for their hospitality during the course of this project: The Mathematics Institute of the University of Warwick, England; I.H.E.S., Fpance; the Mathelatics Department of UC Berkeley, USA; and C.I.M.A.T., Mexico.

\section{References}

[A-B] M.F. Atiyah and R. Bott, The Yang-Mills equations over Riemann surfaces, Philos. Trans. Roy. Soc. London Ser. A 308 (1982) 523-615.

[Be] A. Bertram, Stable pairs and stable parabolic pairs, preprint.

[B-GP] O. Biquard and O. García-Prada, in preparation.

[B1] S.B. Bradlow, Vortices in holomorphic line bundles over closed Kähler manifolds, Commun. Math. Phys. 135 (1990) 1-17.

[B2] S.B. Bradlow, Special metrics and stability for holomorphic bundles with global sections, J. Diff. Geom. 33 (1991) 169-214. 
[B-D1] S.B. Bradlow and G. Daskalopoulos, Moduli of stable pairs for holomorphic bundles over Riemann surfaces, Int. J. Math 2 (1991) 477-513.

[B-D2] S.B. Bradlow and G. Daskalopoulos, Moduli of stable pairs for holomorphic bundles over Riemann surfaces II, Int. J. Math, to appear.

[B-D-W] S.B. Bradlow, G. D. Daskalopoulos and R. A. Wentworth, Birational equivalences of vortex moduli, preprint.

[D1] S.K. Donaldson, Anti-self-dual Yang-Mills connections on a complex algebraic surface and stable vector bundles, Proc. Lond. Math. Soc. 3 (1985) 1-26.

[D2] S.K. Donaldson, Infinite determinants, stable bundles and curvature, Duke Math. J. 54 (1987) 231-247.

[D-K] S.K. Donaldson and P.B. Kronheimer, The Geometry of Four Manifolds, Oxford Mathematical Monographs, Oxford University Press, 1990.

[GP1] O. García-Prada, A direct existence proof for the vortex equations over a compact Riemann surface, Bull. London Math. Soc., to appear.

[GP2] O. García-Prada, Invariant connections and vortices, Commun. Math. Phys., 156 (1993) 527-546 .

[GP3] O. García-Prada, Dimensional reduction of stable bundles, vortices and stable pairs Int. J. Math, to appear.

[G] D. Gieseker, On moduli of vector bundles on an algebraic surface, Ann. of Math. 106 (1977), 45-60.

[H] N.J. Hitchin, The self-duality equations on a Riemann surface, Proc. Lond. Math. Soc. 55 (1987) 59-126.

[K] A. King, Q-bundles on Riemann surfaces, private communication.

[Ko] S. Kobayashi, Differential Geometry of Complex Vector Bundles, Princeton University Press, New Jersey, 1987.

[L] M. Lübke, Stability of Einstein-Hermitian vector bundles, Manuscripta Mathematica 42 (1983) 245-257.

[M] M. Maruyama, Moduli of stable sheaves I and II, J. Math. Kyoto Univ., 17 (1977) 91-126, and 18 (1978) 557-614.

[N-S] M.S. Narasimhan and C.S. Seshadri, Stable and unitary bundles on a compact Riemann surface, Ann. of Math. 82 (1965) 540-564.

[O-S-S] C. Okonek and M. Schneider and H. Spindler, Vector Bundles on Complex Projective Spaces, Progress in Mathematics 3, Birkhäuser, Boston, 1980. 
[T] M. Thaddeus, Stable pairs, linear systems and the Verlinde formula, MSRI preprint.

[U-Y] K.K. Uhlenbeck and S.T. Yau, On the existence of Hermitian-Yang-Mills connections on stable bundles over compact Kähler manifolds, Comm. Pure and Appl. Math. 39-S (1986) 257-293.

Department of Mathematics, University of Illinois, 273 Altgeld Hall, 1409 W. Green Street, Urbana, IL 61801, USA.

Université de Paris-Sud, Mathématiques, Bâtiment 425, 91405 Orsay, France. 